\title{
MAGNETOSPHERIC ACCRETION IN CLOSE PRE-MAIN-SEQUENCE BINARIES
}

\author{
David R. Ardila ${ }^{1}$, Christopher Jonhs-Krull ${ }^{2}$, Gregory J. Herczeg ${ }^{3}$, Robert D. Mathieu ${ }^{4}$, and \\ AlBerto QUiJANO-VODNIZA 5 \\ ${ }^{1}$ The Aerospace Corporation, M2-266, El Segundo, CA 90245; Formerly: NASA Herschel Science Center, \\ California Institute of Technology, MC 100-22, Pasadena, CA 91125, USA; David.Ardila@aero.org \\ ${ }^{2}$ Department of Physics and Astronomy, Rice University, Houston, TX 77005, USA \\ ${ }^{3}$ The Kavli Institute for Astronomy and Astrophysics, Peking University, Beijing 100871, China \\ ${ }^{4}$ Department of Astronomy, University of Wisconsin-Madison, Madison, WI 53706-1582, USA \\ ${ }^{5}$ University of Nariño Observatory, Pasto, Nario, Colombia \\ Received 2015 April 2; accepted 2015 August 13; published 2015 September 29
}

\begin{abstract}
The transfer of matter between a circumbinary disk and a young binary system remains poorly understood, obscuring the interpretation of accretion indicators. To explore the behavior of these indicators in multiple systems, we have performed the first systematic time-domain study of young binaries in the ultraviolet. We obtained far- and near-ultraviolet $H S T /$ COS spectra of the young spectroscopic binaries DQ Tau and UZ Tau E. Here we focus on the continuum from 2800 to $3200 \AA$ and on the $\mathrm{C}$ Iv doublet $(\lambda \lambda 1548.19,1550.77 \AA)$ as accretion diagnostics. Each system was observed over three or four consecutive binary orbits, at phases $\sim 0,0.2,0.5$, and 0.7 . Those observations are complemented by ground-based U-band measurements. Contrary to model predictions, we do not detect any clear correlation between accretion luminosity and phase. Further, we do not detect any correlation between $\mathrm{C}$ Iv flux and phase. For both stars the appearance of the $\mathrm{C}$ Iv line is similar to that of single Classical $\mathrm{T}$ Tauri Stars (CTTSs), despite the lack of stable long-lived circumstellar disks. However, unlike the case in single CTTSs, the narrow and broad components of the $\mathrm{C}$ IV lines are uncorrelated, and we argue that the narrow component is powered by processes other than accretion, such as flares in the stellar magnetospheres and/or enhanced activity in the upper atmosphere. We find that both stars contribute equally to the narrow component $\mathrm{C}$ Iv flux in DQ Tau, but the primary dominates the narrow component $\mathrm{C}$ IV emission in UZ Tau E. The $\mathrm{C}$ iv broad component flux is correlated with other accretion indicators, suggesting an accretion origin. However, the line is blueshifted, which is inconsistent with its origin in an infall flow close to the star. It is possible that the complicated geometry of the region, as well as turbulence in the shock region, are responsible for the blueshifted line profiles.
\end{abstract}

Key words: binaries: spectroscopic - stars: pre-main sequence - stars: variables: T Tauri, Herbig $\mathrm{Ae} / \mathrm{Be}-$ techniques: spectroscopic - ultraviolet: stars

Supporting material: machine-readable table

\section{INTRODUCTION}

Classical T Tauri Stars (CTTSs) are optically visible, young $(<10 \mathrm{Myr})$ low-mass stars, surrounded by an accretion disk. The interaction between the inner disk and the star regulates the system's angular momentum, wind launching, and the accretion of material onto the star. Accretion onto the stellar surface is mediated by the stellar magnetosphere, which truncates the inner radius of the circumstellar disk $\left(r \sim 0.05 \mathrm{AU}, \sim 5 R_{*}\right)$ and channels the gas from the disk to high stellar latitudes (Bertout 2007).

While this picture is reasonably well-established for single stars, its extension to multi-stellar systems remains uncertain. For close binary systems the stars may be surrounded by a circumbinary disk, in addition to possible circumstellar disks. In these systems, the exchange of material between the multiple disks and the role of the magnetic fields in the accretion process remain poorly understood. Because the fraction of multiple systems in young regions such as Taurus-Auriga may be as large as 70\% (Duchêne \& Kraus 2013), understanding the accretion in these remains an important goal in star formation.

Hydrodynamical models (Artymowicz \& Lubow 1996 AL96; Günther \& Kley 2002) predict that accretion streams filling the circumbinary gap develop as a function of orbital phase, on a pattern that depends on the system's binary parameters. These streams may have been observed in the case of SR 24 (Mayama et al. 2010) and GG Tau A (see Beck et al. 2012 and references therein).

For the close binaries DQ Tau and UZ Tau E, long-term photometric observations reveal periodic changes in brightness, which are interpreted as changes in accretion rate (Mathieu et al. 1997; Jensen et al. 2007). Accretion modulated by the orbital period is indeed one of the main predictions of the AL96 models. On the other hand, extensive photometric monitoring of AK Sco, an equal-mass eccentric binary system analogous to DQ Tau, has failed to reveal any periodic signal in the accretion rate (Alencar et al. 2003).

In spite of what are likely to be very different physical configurations, spectroscopic observations of accretion indicators such as $\mathrm{H} \alpha$ and $\mathrm{Ca}$ II $\mathrm{H} \& \mathrm{~K}$ do not reveal morphological or behavioral differences between single and binary systems. For example, Donati et al. (2011) have used spectropolarimetric observations of the V4046 Sgr binary system to model the stars' magnetic fields and conclude that the dipole components are highly tilted with respect to the rotation axis and perpendicular to each other. The result is a very complex system of interacting magnetic fields. In spite of this, gas accretion diagnostics remain stable around each star for many periods (Stempels \& Gahm 2004).

In this paper we explore the role that binary parameters play on the accretion processes of systems in the CTTSs phase. We 
Table 1

Binary Data

\begin{tabular}{lcc}
\hline \hline & DQ Tau & UZ Tau E \\
\hline$\langle V\rangle(\mathrm{mag})^{\mathrm{a}}$ & $13.5 \pm 0.1$ & $12.6 \pm 0.1$ \\
$\langle U\rangle(\mathrm{mag})^{\mathrm{a}}$ & $15.3 \pm 0.1$ & $13.5 \pm 0.1$ \\
$\mathrm{SpT}, \mathrm{SpT} 2^{\mathrm{b}}$ & $\mathrm{M} 0.6, \mathrm{M} 0.6$ & $\mathrm{M} 1.9, \mathrm{M} 4$ \\
$A v^{\mathrm{b}}$ & 1.4 & 0.9 \\
$P($ days $)$ & $15.8043 \pm 0.0024$ & $19.131 \pm 0.003$ \\
$\gamma\left(\mathrm{km} \mathrm{s}^{-1}\right)$ & $22.4 \pm 0.6$ & $13.9 \pm 0.7$ \\
$e$ & $0.556 \pm 0.018$ & $0.33 \pm 0.04$ \\
$\omega(\mathrm{deg})$ & $230.6 \pm 2.2$ & $239 \pm 9$ \\
$T(\mathrm{JD}-2440000)$ & $9582.54 \pm 0.05$ & $11328.3 \pm 0.5$ \\
$q$ & $0.97 \pm 0.15$ & $0.30 \pm 0.03$ \\
$i(\mathrm{deg})$ & $23 \pm 2$ & $54 \pm 5$ \\
$M_{1}\left(M_{\odot}\right)$ & $0.65 \pm 0.1$ & $1.0 \pm 0.1$ \\
$M_{2}\left(M_{\odot}\right)$ & $0.62 \pm 0.1$ & $0.3 \pm 0.1$ \\
$a_{1} \sin i(\mathrm{AU})$ & $0.026 \pm 0.003$ & $0.037 \pm 0.003$ \\
$a_{2} \sin i(\mathrm{AU})$ & $0.027 \pm 0.003$ & $0.10 \pm 0.01$ \\
\hline
\end{tabular}

Notes. The data for DQ Tau were taken from Mathieu et al. (1997) unless indicated. The data for UZ Tau E were taken from Jensen et al. (2007).

${ }^{a}$ This work.

${ }^{\mathrm{b}}$ Spectral types and extinctions from Herczeg \& Hillenbrand (2014), except for the spectral type of UZ Tau E's secondary, taken from Prato et al. (2002).

have obtained far- and near-ultraviolet (FUV and NUV) highresolution spectra of the well-studied binaries DQ Tau and UZ Tau E (See Table 1) over multiple contiguous binary periods, using the Cosmic Origins Spectrograph (COS) on the Hubble Space Telescope (HST). This is the first time-domain study with high-resolution UV spectroscopy for CTTSs. We complement the HST/COS spectroscopic observations with high cadence, ground-based optical photometry.

Here we focus on the NUV continuum from 2800 to $3200 \AA$ and the $\mathrm{C}$ IV doublet $(\lambda \lambda 1548.19,1550.77)$ as accretion diagnostics. The NUV continuum provides a straightforward measure of the accretion luminosity to the system. The bulk of the $\mathrm{C}$ IV line is believed to originate as the accretion stream crashes into the stellar surface (Lamzin 1995; Günther \& Schmitt 2008). As such, it tracks a more compact region than $\mathrm{H} \alpha$. In addition, the recent study by Ardila et al. (2013; A13) provides a large sample of $\mathrm{C}$ IV line observations in single and multiple systems that serves to provide context to the observations presented here.

We describe the observations in Section 3. In Section 4, we calculate the accretion luminosity as a function of phase. We then study the behavior and general shape of the $C$ IV lines in Section 5. In Section 6 we address the origin of the emission and the issue of determining accretion to individual stars. Further discussion and conclusions are provided in Section 7.

\section{THE TARGETS}

Table 1 shows the parameters adopted in this paper for DQ Tau and UZ Tau E. Both systems are young, eccentric spectroscopic binaries, with UZ Tau E being brighter than DQ Tau in the optical and the ultraviolet. Dynamically, the main difference between the systems is the secondary-to-primary mass ratio $(q)$, which is 1 for DQ Tau but 0.3 for UZ Tau E. We assume a mean distance of $140 \mathrm{pc}$ to the targets. Distance measurements within Taurus show a spread of \pm 15 pc (Loinard et al. 2007; Torres et al. 2009).
UZ Tau is a quadruple system, with UZ Tau Eab (UZ Tau Aab) 3". 7 away from UZ Tau Wab (UZ Tau Bab-Dommanget \& Nys 2002). In turn, UZ Tau E is a single-lined spectroscopic binary (Mathieu et al. 1996), with the lines from the secondary detected only in NIR spectra (Prato et al. 2002).

The mass of the UZ Tau E primary, as derived from evolutionary tracks, is about $2 \times$ smaller than the dynamical mass, which may indicate that the target distance is smaller than the assumed $140 \mathrm{pc}$ (Prato et al. 2002).

The circumbinary disk has been resolved at a variety of wavelengths (e.g., Dutrey et al. 1996; Jensen et al. 1996; Simon et al. 2001). Observations of the dust continuum at $880 \mu \mathrm{m}$ reveal a circumbinary disk $219 \mathrm{AU}$ in radius (Harris et al. 2012), coplanar with the binary orbit (Jensen et al. 2007).

As a system, UZ Tau E shows evidence of ongoing accretion in the form of strong $\mathrm{H} \alpha$ emission (Martín et al. 2005) and a [O I] jet (Hartigan et al. 2004). Jensen et al. (2007) show that BVRI photometry of the UZ Tau E system exhibits significant random variability, but with a periodic pattern consisting of a broad peak in enhanced brightness. The period is roughly consistent with the binary period, as expected from the AL96 models. All four bands show a gradual increase in brightness over the 3 years of observations. A periodic signal is not observed in the $\mathrm{H} \alpha$ equivalent width observations.

DQ Tau was identified as a double-lined spectroscopic binary by Mathieu et al. (1997). The circumbinary disk is weakly resolved at $1.3 \mathrm{~mm}$ by Guilloteau et al. (2011). Observationally, the gap cleared by the stars is not empty, at least not all the time. Understanding the spectral energy distribution requires a small amount of hot inner gap material in order to reproduce the infrared excess (Mathieu et al. 1997). Carr et al. (2001) detect CO 1-0 and 2-1 emission lines with kinematic and thermal characteristics consistent with an origin within $0.5 \mathrm{AU}$ and interferometric observations by Boden et al. (2009) have identified K-band excess at distance scales $\sim 0.1-0.2$ AU.

Mathieu et al. (1997) show that, occasionally, the system becomes brighter (by about $1 \mathrm{mag}$ in $V$ ) and bluer around periastron passage. Traditional CTTSs accretion diagnostics also become stronger near some of the periastron passages (Basri et al. 1997). Continuum excess and strong $\mathrm{H} \alpha$ emission are observed at all phases, indicating that accretion happens throughout the binary orbit (Basri et al. 1997).

For UZ Tau E, the measured rotational speed of the primary is $v \sin i=16 \mathrm{~km} \mathrm{~s}^{-1}$, which implies a rotational period of 4.9 days (Jensen et al. 2007). The rotational velocity of the secondary is unknown. For DQ Tau, both stars have similar $v \sin i=10 \pm 2 \mathrm{~km} \mathrm{~s}^{-1}$, which implies $P_{\text {Rot }}=3$ days (Basri et al. 1997). In both cases the determination of the rotational period assume that the inclination of the orbit and that of the stellar rotation axis is the same.

In this paper the stars are observed at phases $\sim 0, \sim 0.2$, $\sim 0.5$, and $\sim 0.7$ (Section 3 ). Errors in the period determination coupled with the long time since the reference periastron passage result in phase errors of \pm 0.06 and \pm 0.04 for DQ Tau and UZ Tau E, respectively. For DQ Tau, a 0.05 error in the phase at periastron corresponds to $\sim 70^{\circ}$ uncertainty in the position of the targets compared to $\sim 7^{\circ}$ uncertainty at apastron. For UZ Tau E, the same phase error corresponds to $10^{\circ}$ uncertainty at apastron and $40^{\circ}$ uncertainty at periastron.

A range of extinctions is available from the literature for each star. For DQ Tau, values range from $A_{v}=0.71 \mathrm{mag}$ 
(Gullbring et al. 1998) to $A_{v}=2.52 \mathrm{mag}$ (Furlan et al. 2011). For UZ Tau E, possible values include $A_{v}=0.5 \mathrm{mag}$ (Ingleby et al. 2009) and $A_{v}=1.49 \mathrm{mag}$ (Kenyon \& Hartmann 1995). The measured fluxes are very sensitive to the choice of extinction adopted. For DQ Tau and UZ Tau E, we adopt $A_{v}=1.4 \mathrm{mag}$ and $A_{v}=0.9 \mathrm{mag}$, respectively, from Herczeg \& Hillenbrand (2014). These were determined consistently from optical measurements within a large number of young stars, taking into account the presence of an accretion continuum.

\subsection{Are there Circumstellar Disks?}

As we have mentioned, previous observations show that gas resides in the circumbinary gap for both UZ Tau E and DQ Tau. However, as we show below, it is unlikely that this gas forms stable, long-lived circumstellar disks. This is because potential circumstellar disks have their inner radius truncated by the magnetosphere and the outer radius truncated by dynamical effects between the stars. With the possible exception of a narrow disk around the UZ Tau E primary, there is not enough space between the stars for a disk to survive long-term.

For DQ Tau, the separation between the binary members ranges from 13 to $45 R_{\odot}$. Considering only gravitational forces and ignoring radiation or hydrodynamic effects, the largest stable, long-lived circumstellar disks should be smaller than $\sim 3 R_{\odot}$ (Holman \& Wiegert 1999). However, the observational indicators mentioned in the introduction, as well as the shape of the $\mathrm{C}$ IV lines we present in this paper, point to the fact that accretion to the stars is magnetospherically dominated. The typical magnetosphere radius in single CTTSs is $\sim 5 R_{*}$. In the case of the DQ Tau system, and assuming that each star has a radius of $1.6 R_{\odot}$ (Cox 2000), the magnetophere radius will be 8 $R_{\odot}$. Because the magnetosphere is larger than the largest stable disk around each star, it is unlikely that such circumstellar disks exist. In addition, the DQ Tau magnetospheres will interact with each other at periastron, likely redistributing the accretion material among the stars.

For UZ Tau E, the separation between the stars ranges from 24 to $48 R_{\odot}$. Again, the largest circumstellar disks have radii of $9 R_{\odot}$ around the primary or $4 R_{\odot}$ around the secondary (Holman \& Wiegert 1999). In UZ Tau E, the stellar magnetospheres have radii of 10 and $8 R_{\odot}$ for the primary and secondary, assuming $\sim 5 R_{*}$ with $R_{1}=1.9 R_{\odot}$ and $R_{2}=1.6 R_{\odot}$ (Cox 2000). For the primary, the radius of the largest possible circumstellar disk is comparable to the radius of the magnetosphere. If there is a stable disk present, it will look like a narrow ring. For the secondary, a possible circumstellar disk would be pushed out by the magnetosphere, beyond the region of stability.

Therefore, there may be a narrow stable ring of material around the primary of UZ Tau E. For the rest of the stars in the systems there is no long-lived circumstellar disk to serve as source for the accretion material. Any gas in the gap resides in dynamically unstable structures and is continuously replenished from the circumbinary disk.

\section{OBSERVATIONS}

\subsection{HST Observations}

DQ Tau was observed with $H S T / C O S$ four times per binary orbit, during three consecutive binary orbits, at phases $\sim 0$, $\sim 0.2, \sim 0.5$, and $\sim 0.7$. The original experimental design called
Table 2

$H S T /$ COS Observation Log

\begin{tabular}{lcccc}
\hline \hline Visit $^{\text {a }}$ & Target & Date (UT) & Time (UT) & Phase $^{\text {b }}$ \\
\hline 1 & UZ Tau E & 2011 Feb 2 & $14: 19: 39$ & 0.03 \\
2 & UZ Tau E & 2011 Feb 6 & $3: 47: 41$ & 0.22 \\
3 & UZ Tau E & 2011 Feb 11 & $14: 29: 49$ & 0.50 \\
4 & UZ Tau E & 2011 Feb 16 & $0: 15: 42$ & 0.73 \\
5 & UZ Tau E & 2011 Feb 21 & $5: 45: 15$ & 1.01 \\
6 & UZ Tau E & 2011 Feb 25 & $7: 11: 08$ & 1.22 \\
7 & UZ Tau E & 2011 Mar 3 & $2: 08: 57$ & 1.52 \\
$8^{\text {c }}$ & UZ Tau E & 2011 Mar 6 & $22: 40: 33$ & 1.72 \\
$9^{\mathrm{d}}$ & UZ Tau E & 2011 Mar 12 & $22: 35: 17$ & 2.03 \\
10 & UZ Tau E & 2011 Mar 16 & $4: 48: 25$ & 2.21 \\
11 & UZ Tau E & 2011 Mar 22 & $12: 24: 53$ & 2.54 \\
12 & UZ Tau E & 2011 Mar 25 & $23: 37: 09$ & 2.72 \\
$13^{\mathrm{e}}$ & DQ Tau & 2011 Feb 11 & $12: 34: 49$ & 0.00 \\
14 & DQ Tau & 2011 Feb 15 & $5: 52: 13$ & 0.24 \\
15 & DQ Tau & 2011 Feb 19 & $23: 27: 36$ & 0.54 \\
16 & DQ Tau & 2011 Feb 22 & $14: 03: 29$ & 0.70 \\
17 & DQ Tau & 2011 Feb 27 & $18: 20: 47$ & 1.03 \\
18 & DQ Tau & 2011 Mar 3 & $0: 26: 38$ & 1.24 \\
19 & DQ Tau & 2011 Mar 7 & $8: 27: 25$ & 1.51 \\
20 & DQ Tau & 2011 Mar 10 & $8: 57: 32$ & 1.70 \\
21 & DQ Tau & 2011 Mar 15 & $17: 43: 06$ & 2.04 \\
22 & DQ Tau & 2011 Mar 18 & $8: 00: 31$ & 2.21 \\
23 & DQ Tau & 2011 Mar 23 & $7: 48: 04$ & 2.52 \\
24 & DQ Tau & 2011 Mar 26 & $13: 54: 06$ & 2.73 \\
$26^{\mathrm{f}}$ & UZ Tau E & 2011 Apr 1 & $1: 01: 05$ & 3.03 \\
$25^{\mathrm{f}}$ & UZ Tau E & 2011 Apr 10 & $7: 09: 44$ & 3.52 \\
\hline
\end{tabular}

Notes.

${ }^{\text {a }}$ Each visit corresponds to one $H S T$ orbit. For each visit, the target was acquired and then observed with the COS G230L grating ( $\sim 200 \mathrm{~s})$ followed by the COS G160M grating ( $\sim 1800 \mathrm{~s}$ ).

b Binary orbital phase at the beginning of the observation.

${ }^{\mathrm{c}}$ FGS lost lock on the guide stars. No data taken in G230L; The G160M observations are nominal.

${ }^{\mathrm{d}}$ Shutter closed in acquisition, FGS lost lock on the guide stars. No data taken. e Shutter closed during acquisition. Only G160M segment B observations are nominal.

${ }^{\mathrm{f}}$ Rescheduling of visits 08 and 09.

for observations of UZ Tau E with the same cadence. However, the NUV observations at phase $\sim 0.7$ in the second orbit and both the FUV and NUV observations at phase $\sim 0$ in the third orbit failed. They were replaced by observations at phases $\sim 0$ and $\sim 0.5$ in a fourth binary orbit. Table 2 shows the log of HST observations. See also Figure 1.

Each $H S T / \mathrm{COS}$ observation lasted for a single $H S T$ orbit. For each observation, the target star was acquired using the ACQ/IMAGE acquisition mode with the MIRRRORB spectral element. The target was then observed with the G230L and G160M gratings. The G230L grating was used in the $2950 \AA$ setting, covering from 1650 to $2050 \AA$ (Stripe A), and from 2750 to $3150 \AA$ (Stripe B). The G160M grating was used in the central wavelength setting $1577 \AA$, which covers from 1386 to $1599 \AA$ (Segment $\quad$ B), and from 1577 to $1751 \AA$ (Segment A). For G160M we obtained two equal length time-tagged spectra per observation, at (Fixed-Pattern Position) FP-POS $=3$ and FP-POS $=4$. See Massa (2013).

The G160M spectra provide a velocity resolution of $\Delta v \sim 17 \mathrm{~km} \mathrm{~s}^{-1}(R=16,000-21,000)$ with seven pixels per resolution element, while the G230L spectra provide a 


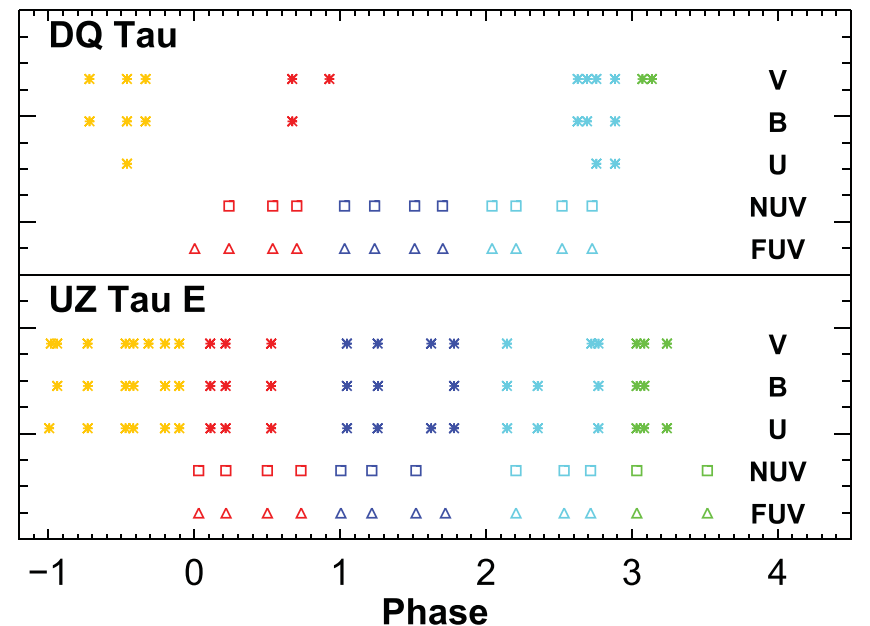

Figure 1. Timeline for $H S T$ spectroscopy and ground-based optical photometry. The systematic errors in the phase determination are \pm 0.06 and \pm 0.04 for DQ Tau and UZ Tau E, respectively. V and B observations are not discussed in this paper.

resolution of $\Delta v \sim 120 \mathrm{~km} \mathrm{~s}^{-1}(R=2100-3200)$ (Osterman et al. 2011; Green et al. 2012).

For UZ Tau E, each G230L observation was 200 s long, and each G160M observation was $1944.3 \mathrm{~s}$ long. The fainter DQ Tau needed longer acquisition times, resulting in shorter spectroscopic observations. For DQ Tau, observations with the G230L grating were exposed for $192 \mathrm{~s}$, while those with G160M were exposed for $1744.2 \mathrm{~s}$.

We obtained one-dimensional, co-added spectra using the COS calibration pipeline (CALCOS) with alignment and coaddition obtained using the IDL routines described by Danforth et al. (2010). Some of the science in this paper depends on the accuracy in the determinations of line centroids, in particular for observations taken with the G160M grating. The absolute wavelength scale accuracy is $\sim 15 \mathrm{~km} \mathrm{~s}^{-1}(1 \sigma)$. $50 \%$ of this is allocated to errors in the target acquisition, while the other $50 \%$ is due to geometric distortion in the FUV detectors.

The acquisition images provide a direct way to correct for the errors in the stellar centering. We compare the position of the center of the aperture (as given by the ACQPREFX/Y keywords) with the target centroid in the acquisition images. Excluding visits 9 and 13 for which the shutter was closed during acquisition, we find average differences of $0 . " 012$ and 0 " 027 in the dispersion and cross-dispersion directions, respectively. In the dispersion direction, this difference corresponds approximately to one tenth of the resolution element, resulting in systematic velocity shifts of $\sim 2 \mathrm{~km} \mathrm{~s}^{-1}$. Evaluation of the COS FUV and NUV spectroscopic performance indicates that the offsets are not large enough to degrade the throughput (Goudfrooij et al. 2010; Ghavamian et al. 2010).

Based on this analysis, we correct each centroid velocity by a value given by the shift determined above. The residual systematic error is assumed to be $\sim 7.5 \mathrm{~km} \mathrm{~s}^{-1}$, as suggested by the instrument documentation. In this paper we only work with $\mathrm{C}$ IV lines, which are always positioned in a similar region of the detector. Therefore, the effect of the residual systematic error is an overall shift in all the data. We do not include it in our analysis.

Table 2 shows that some observations failed due to instrumental problems. For visit $8 \mathrm{UZ}$ Tau E was acquired
Table 3

University of Nariño Observatory-Observation Log

\begin{tabular}{lccccc}
\hline \hline Target & $\begin{array}{c}\text { Date, Time } \\
(\mathrm{UT})\end{array}$ & Band & $\begin{array}{c}\text { Exp. } \\
(\text { Sec. })\end{array}$ & Mag. ${ }^{\text {a }}$ & Phase \\
\hline DQ Tau & $12 / 6 / 10,2: 15: 02$ & $\mathrm{~B}$ & 120 & 13.7 & -4.26 \\
DQ Tau & $12 / 6 / 10,2: 20: 31$ & $\mathrm{~V}$ & 300 & 12.7 & -4.26 \\
DQ Tau & $12 / 28 / 10,2: 05: 34$ & $\mathrm{~B}$ & 90 & 15.1 & -2.87 \\
\hline
\end{tabular}

Note.

a The magnitude uncertainty is $\sim 0.1 \mathrm{mag}$.

(This table is available in its entirety in machine-readable form.)

correctly but the Fine Guidance Sensor (FGS) lost the lock on guide stars during the NUV observations. For visit 9, the shutter was closed during acquisition and the FGS lost the lock on the guide stars, resulting in failed NUV and FUV observations.

For the DQ Tau observation in visit 13, the shutter was closed during acquisition, but the FGS kept a lock on the guide stars. In other words, the observation was done on the telescope's blind pointing. The resulting FUV segment A and segment B spectra are 0." 3 and 0." 08 away, respectively, from the nominal cross-dispersion position. If we assume that the offset is the same in the dispersion direction, this corresponds to $2 \mathrm{x}$ and $0.06 \mathrm{x}$ the size of the spectral resolution element, equivalent to $\sim 35 \mathrm{~km} \mathrm{~s}^{-1}$ and $\sim 1 \mathrm{~km} \mathrm{~s}^{-1}$, respectively. For segment $\mathrm{B}$, this offset is within the observed range of offsets for other observations.

Inspection of the actual data for visit 13 shows that the emission lines in segment B (1386-1599 $\AA$ ) are typical in shape and flux when compared to other DQ Tau observations. However, observations of $\mathrm{H}_{2}$ within segment A (1577-1751 $\AA$ ) do show large blueshifts when compared to segment B $\mathrm{H}_{2}$ observations. We do not see these offsets between the A and B segments in other DQ Tau observations nor in the UZ Tau E observations. Surprisingly, the NUV spectra do not seem anomalously weak, in spite of the large offset.

Based on this analysis, for visit 13 we will only use the segment B observations, and disregard segment $\mathrm{A}$ and the NUV observations. We do not correct the segment B data by the pointing error.

\subsection{Ground-based Observations}

We obtained contemporaneous ground-based $U B V$ photometry with the $14^{\prime \prime}$ telescope from the University of Nariño Observatory, optical spectroscopy with the Sandiford Echelle Spectrometer on the $2.1 \mathrm{~m}$ Otto Struve Telescope at McDonald Observatory, near-infrared spectroscopy with the CSHELL spectrograph on the NASA Infrared Telescope Facility, and near-infrared spectroscopy with GNIRS instrument on Gemini North. In this paper we focus on the U-band photometry only.

The University of Nariño Observatory $\left(01^{\circ} 12^{\prime} 44^{\prime \prime} \mathrm{N} ; 77^{\circ}\right.$ $17^{\prime} 27^{\prime \prime} \mathrm{W}, 2500$ MASL) is located in Pasto, Colombia. The observing $\log$ for the observations is in Table 3. $U B V$ observations were obtained before and during the $H S T$ campaign and reduced in the standard way. The distribution of $U B V$ photomery values is consistent with that obtained using the catalog from Herbst et al. (1994). 

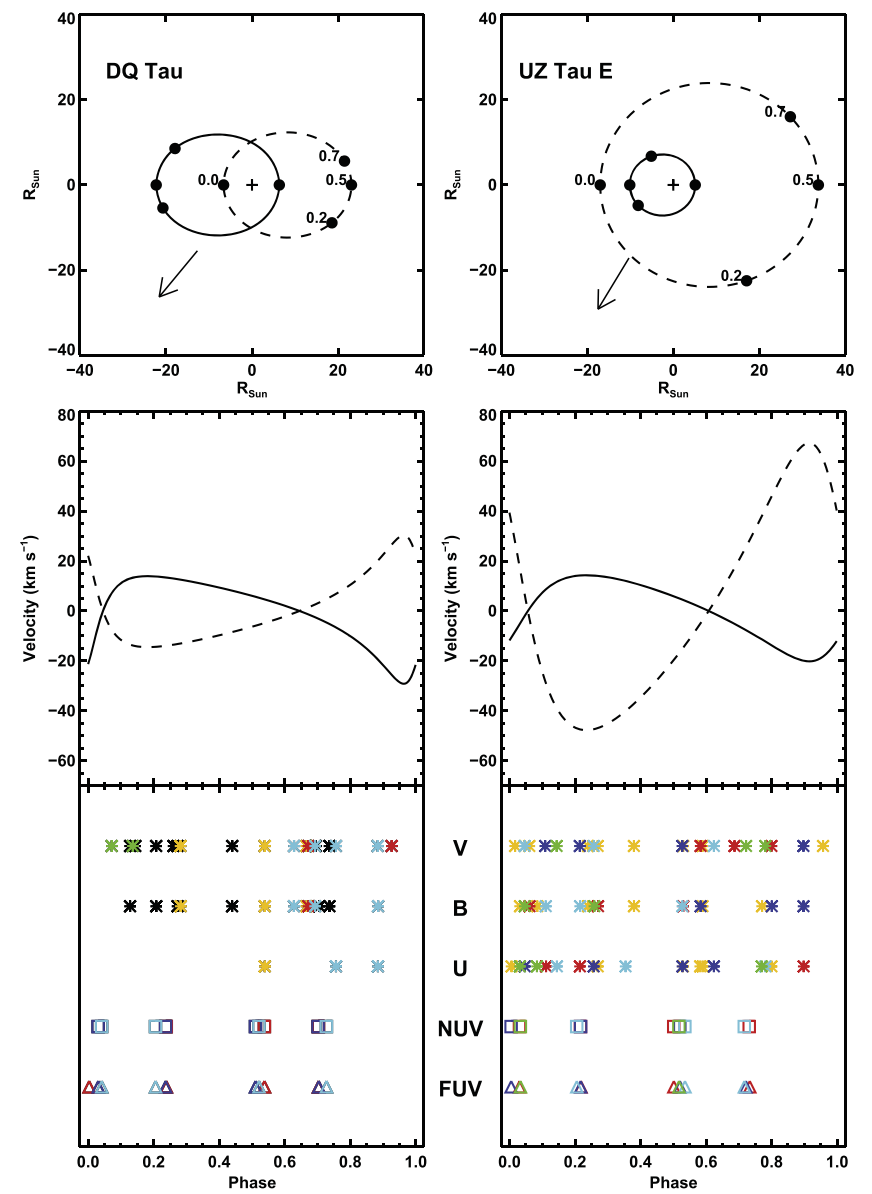

Figure 2. Summary of the observations considered in this paper. Left: DQ Tau; Right: UZ Tau E. Top: top view of the binary configuration. The Earth is along the direction of the arrow but off the paper plane $\left(70^{\circ}\right.$ for DQ Tau, $40^{\circ}$ for UZ Tau E). The nominal phases of the HST's observations are indicated. Middle: observed radial velocity as a function of phase, reconstructed from the binary parameters. Bottom: observations as a function of phase, folded to one period The different colors indicate observations taken in different orbital periods.

Figure 2 summarizes the data coverage as a function of phase.

\section{ACCRETION LUMINOSITY AS A FUNCTION OF ORBITAL PHASE}

In order to explore the behavior of the accretion luminosity as a function of phase we make use of three data sets: archival data in the U-band, our own ground-based U-band data, and the NUV continuum measurements from HST. The result is shown in Figure 3.

To estimate the accretion luminosity from the ground-based photometric data we have used the relationship between excess U-band luminosity $\left(L_{\mathrm{U}}\right)$ and accretion luminosity from Gullbring et al. (1998). For each star, we assume an intrinsic U-band luminosity $\left(L_{\mathrm{U}}=0.004 L_{\odot}\right.$ for DQ Tau, $L_{\mathrm{U}}=0.002$ $L_{\odot}$ for UZ Tau E) based on the spectral types and radii of the systems and subtract this from the measured U-band luminosity. The typical measured U-band luminosities for these objects are $\sim 5 \times$ to $10 \times$ larger than their intrinsic ones.

In CTTSs, the NUV continuum is believed to originate at the end of the accretion flow and it serves as a proxy for the accretion luminosity. The estimate accretion luminosity from NUV continuum measurements requires that we translate the

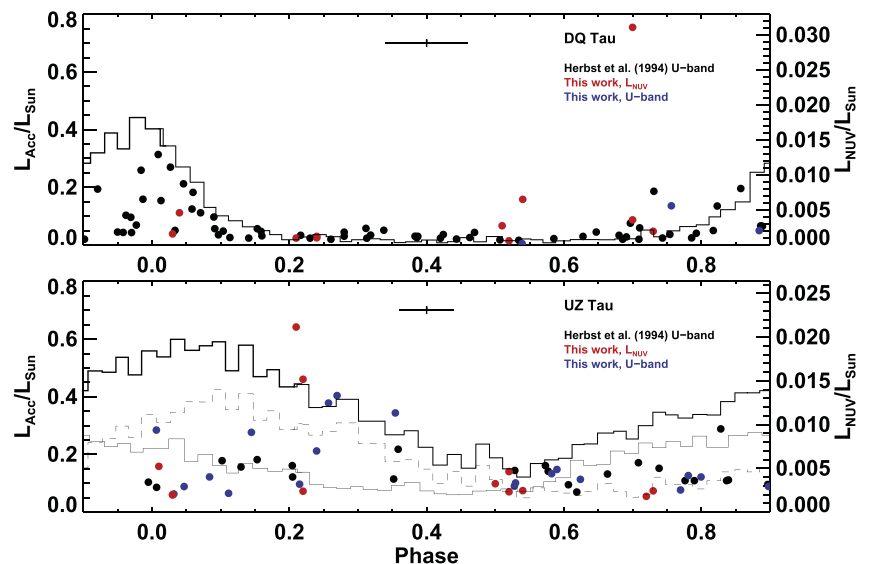

Figure 3. Accretion luminosity vs. phase. The horizontal error bars represent the error in the phase determination. The black (error $\pm 2 \%$ ) and blue (error $\pm 10 \%)$ points are based on photometric U-band measurements from Herbst et al. (1994) and this paper, respectively. For each measurement, we estimate the excess U-band luminosity and use it to obtain $L_{\text {Acc }}$ (Gullbring et al. 1998). The red points (error $\pm 3 \%$ ) are based on our HST NUV observations converted to U-band excess luminosity and then to accretion luminosity. The measured $L_{\mathrm{NUV}}$ are indicated in the right axis. The histogram line shows the AL96 models that most approximate the DQ Tau and UZ Tau E, scaled vertically by an arbitrary amount. For UZ Tau E we plot the contribution of the primary (dashed line), the secondary (solid line), and both (thick solid line).

spectroscopic measurements to excess U-band luminosities. We measure the NUV luminosity ( $\left.L_{\mathrm{NUV}}\right)$ by integrating the observed flux from 2776 to $3176 \mathrm{~A}$, avoiding the $\mathrm{Mg}$ II line between 2820 and $3170 \AA$. As Ardila et al. (2002) show, the $\mathrm{Mg}$ II line is likely formed in the extended accretion magnetosphere(s), while the NUV continuum is dominated by emission from the bottom of the accretion shock.

For DQ Tau we derive a scaling factor between NUV luminosity and $L_{\mathrm{U}}$ from $H S T$ 's observations in visit 24 (on $3 /$ 26/11, 13:54:06) and the ground-based U-band observations on 3/27/11, 1:00:00. We use this same scaling factor for all NUV observations. For UZ Tau E, we interpolate the U-band measurements to determine $L_{\mathrm{U}}$ at each point in time in which $L_{\mathrm{NUV}}$ was obtained and obtain a mean multiplicative factor to convert between the observed NUV luminosity and the U-band luminosity.

In this way, we can associate a U-band luminosity with each NUV measurement. We then subtract the assumed intrinsic U-band luminosity and use the correlation from Gullbring et al. (1998) to obtain the accretion luminosity. The result is an almost linear relationship between $L_{\mathrm{NUV}}$ and $L_{\mathrm{Acc}}$.

Figure 3 shows the behavior of the accretion luminosity with phase, both for literature measurements (Herbst et al. 1994) and for the observations obtained in this work. The random errors in the accretion luminosity due to measurement errors range from $2 \%$ to $10 \%$. However, the calculation is dominated by the systematic error in the fit for the relationship between $L_{\mathrm{U}}$ and $L_{\text {Acc }}$ (Gullbring et al. 1998). For our data, this translates into systematic errors in the accretion luminosity of $50 \%$ for DQ Tau, and $30 \%$ for UZ Tau E.

To compare these observations with predictions, we turn to the models from AL96. The parameters in these are not exactly the same as in our systems but they serve as useful comparisons with the data presented here.

In both cases we have converted the predicted accretion rates from the models to predicted accretion luminosities. Figure 3 
shows the predicted behavior of accretion luminosity with phase for $M_{2} / M_{1}=0.8, e=0.5$ (top, approximating DQ Tau), and $M_{2} / M_{1}=0.4, e=0.1$ (bottom, approximating UZ Tau E). Following Jensen et al. (2007) we have shifted the bottom model by +0.2 in phase to account for the difference in eccentricity between the model and UZ Tau.

For DQ Tau, we observe a high accretion point at phase 0.7 not observed in any other orbit. The accretion luminosity at this phase is $\sim 3 \times$ larger than the next larger value, and $\sim 10 \times$ larger than the average without it. We do not see a correlation between accretion luminosities determined spectroscopically and phase.

Accretion luminosities calculated based on Herbst et al. (1994) data (taken between 1981 and 1986) do show a peak at a phase close to zero. Errors in the period determination of the binary result in a systematic uncertainty of $\sim 0.05$ (one tick in the plot) for those observations. Such a shift would match the model and the data. The average increase of accretion luminosity at periastron is also observed in the VRI photometric measurements by Mathieu et al. (1997).

For UZ Tau E we also observe large accretion luminosity excursions within the same phases at different binary orbits. Jensen et al. (2007) noted that there is a minimum I-band brightness between phases 0.4 and 0.6 and broad maximum centered at phase $\sim 0$. We do not observe such a pattern in the $L_{\text {Acc }}$ measurements presented here.

Overall, the spectroscopic observations presented here do not provide further support for the idea that accretion is phasedependent.

\section{THE BEHAVIOR OF THE C IV LINES}

As mentioned in the introduction, the $\mathrm{C}$ IV doublet lines track the accretion spot, as they are believed to form close to the stellar surface, where the magnetospheric stream encounters the stellar chromosphere. For the current data set, the $\mathrm{C}$ IV line observations are shown in Figures 4 and 5. For both systems, the appearance of the line is that of a narrow component on top of a broad one, with all the peaks at positive velocities. This is the typical morphology of the C Iv lines for CTTSs (A13)

Also visible is the molecular hydrogen line $R(3) 1-8$ at $-167 \mathrm{~km} \mathrm{~s}^{-1}$, which overlaps with the blue wing of the $1548 \AA \mathrm{C}$ Iv line. For DQ Tau, the flux in this line is small compared to the $\mathrm{C}_{\mathrm{IV}}$, but this is not the case for UZ Tau E, where it significantly modifies the $\mathrm{C}$ IV line profile.

The $\mathrm{C}$ IV lines never appear narrow and symmetric, as observed in Weak T Tauri Stars (WTTSs). For those nonaccreting systems, C IV emission is dominated by the upper stellar atmosphere, the transition region. The very broad appearance of the $\mathrm{C}$ IV lines in DQ Tau and UZ Tau E indicates that accretion happens continuously throughout the orbit. In turn, this suggests the presence of a gas reservoir around the stars, either long-lived or continuously replenished. The same conclusion was reached by Basri et al. (1997) on the basis of optical emission-line observations.

The right column of Figure 4 shows all the DQ Tau $\mathrm{C}$ IV profiles within a given orbit, overplotted. From orbit to orbit we observe large average changes. The bottom row shows all profiles with similar phases. While all the $\Phi \sim 0$ and $\Phi \sim 0.2$ observations are similar to each other in shape and flux, the $\Phi \sim 0.5$ and $\Phi \sim 0.7$ observations show variations of a factor of $\sim 4$ in line flux.
For UZ Tau E (Figure 5), the situation is similar. The flux is almost constant during the first orbit, but shows large variations, also by factors of $\sim 4$, during the second and third orbits. In terms of the phase, we observe a larger scatter at $\Phi \sim 0.2$ than at $\Phi \sim 0.5$.

Figure 6 shows that the variability in the C Iv lines is dominated by variability in the lines' narrow core components. These change much more than the wings of the line for the observations presented here.

To measure the $\mathrm{C}$ IV flux we follow the procedure described in A13, subtracting the continuum, and integrating from -500 to $+1000 \mathrm{~km} \mathrm{~s}^{-1}$ after interpolating over the $\mathrm{H}_{2} R(3) 1-8$ line. The results of the measurements are listed in Table 4.

Figure 7 (Top) shows the measured $\mathrm{C}$ IV flux as a function of phase. The errors in the $\mathrm{C}$ IV luminosity are between $1 \%$ and $3 \%$. For DQ Tau the flux at phase $=0.2$ is the smallest in average $\left(L_{\mathrm{CIV}} / L_{\odot}=2.6 \pm 0.7 \times 10^{-4}\right)$ while that at phase $=0$ is the largest $\left(L_{\mathrm{CIV}} / L_{\odot}=4.6 \pm 0.5 \times 10^{-4}\right)$. For UZ Tau E, the largest average flux occurs at phase $=0.2\left(L_{\mathrm{CIV}} / L_{\odot}=3.3 \pm\right.$ $\left.1.9 \times 10^{-4}\right)$, while the smallest occurs at phase $=0.7\left(L_{\mathrm{CIV}} /\right.$ $\left.L_{\odot}=1.1 \pm 0.4 \times 10^{-4}\right)$. No correlation with phase is observed in the overall sample.

As in other CTTSs, accretion and C IV luminosities are well correlated, as seen in Figure 7 (bottom). Yang et al. (2012) derived a correlation $\left(L_{\mathrm{C} \text { IV }} \propto L_{\mathrm{Acc}}^{0.9}\right)$ for these two quantities based on a large sample of CTTSs (not screened for multiplicity). In that work, accretion luminosities are obtained from the literature, and represent determinations by many different independent methods. DQ Tau was not included in their sample but UZ Tau E was, with $A_{V}=0.3 \mathrm{mag}$.

Neither the DQ Tau nor UZ Tau E observations we obtain here exactly match the correlation from Yang et al. (2012) but this is not very significant, as the scatter in that correlation is 0.6 dex. As we mentioned before, the error in the accretion luminosity is dominated by the systematic error in the translation from $L_{\mathrm{U}}$ to $L_{\mathrm{Acc}}$, and it may be as large as $50 \%$.

Because DQ Tau is an equal mass binary, it is not unreasonable to assume that both stars contribute equally to the accretion luminosity and C IV line. Figure 7 (bottom) also shows the emission from each star under this assumption. The result still does not match the correlation, although a smaller extinction would bring the DQ Tau data closer to the solid line. For UZ Tau E, the observed accretion luminosities should be dominated by one or the other of the binary members, depending on the phase.

Note that the very large accretion luminosity point in DQ Tau shown in Figure $3(5 \times$ larger than the next largest point) occurs simultaneously with the largest C IV emission (20\% larger than the next largest point), although the increase is substantially smaller than predicted by the approximately linear correlation from Yang et al. (2012).

\section{ANALYSIS}

\subsection{Comparison to Single CTTSs}

As discussed in A13, the narrow and broad components of the $\mathrm{C}$ IV line may form in different regions of the accretion flow. To explore the contributions of each region in each star to the observed line, we follow the same procedure as in A13 and decompose each $\mathrm{C}$ Iv line in narrow and broad Gaussian components (NCs and BCs). For each doublet, the decomposition assumes that the spectral distance between the lines is 

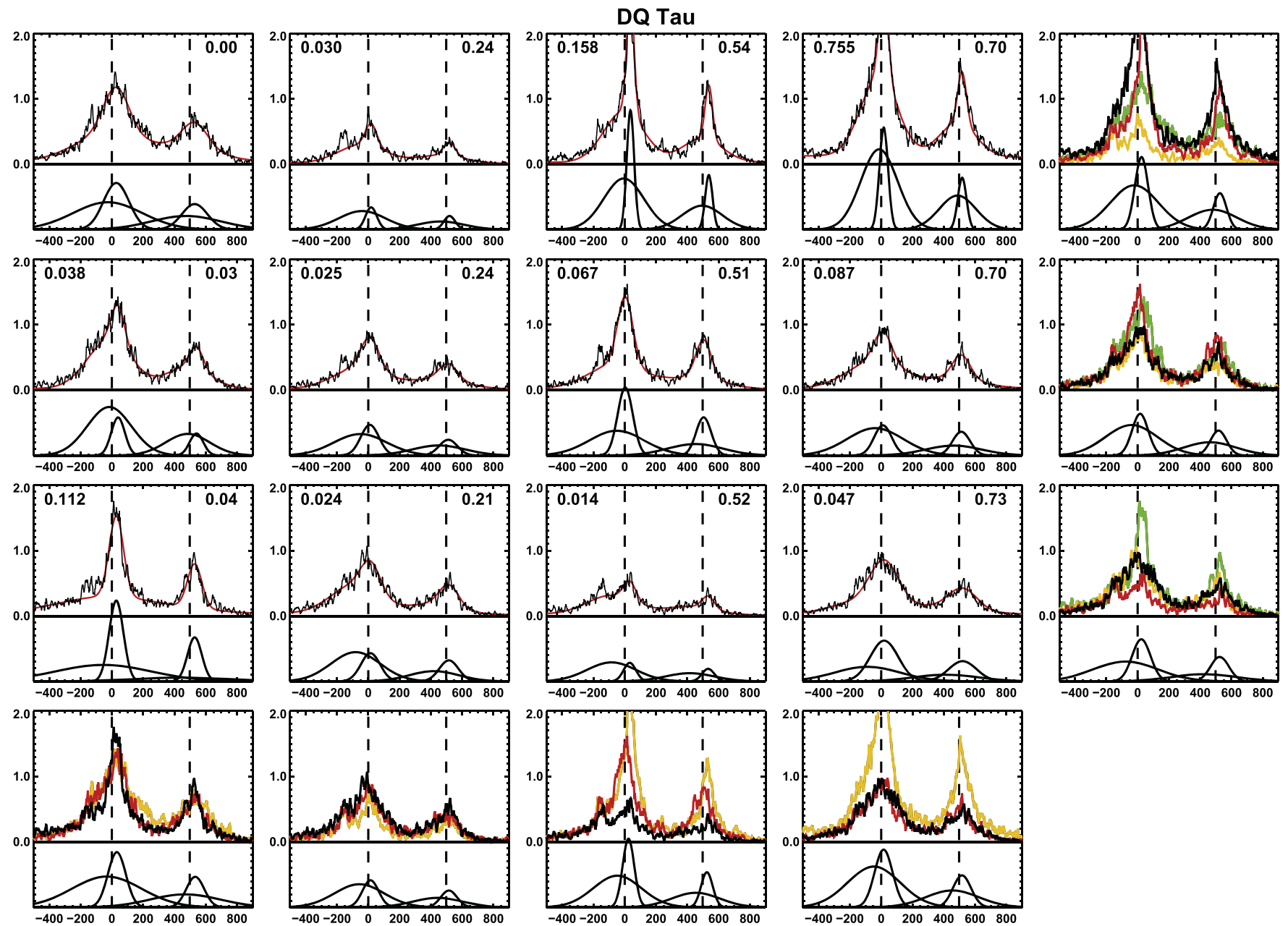

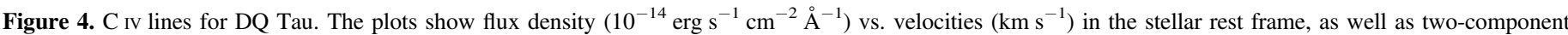

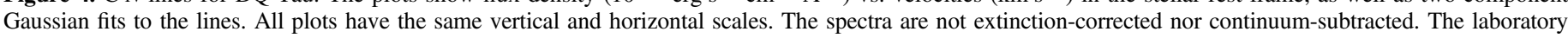

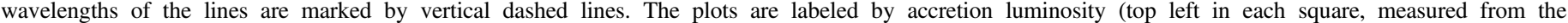

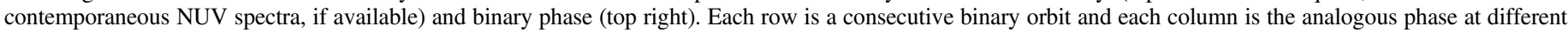

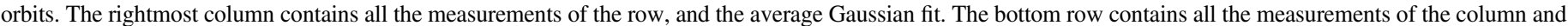
the average Gaussian fit.

constant and that the NCs and BCs have the same width and velocity in both members of the doublet. The results of this decomposition are shown in Figures 4 and 5 and the Gaussian parameters are listed in Table 4.

The table also lists the average values for the fitted and measured parameters. We compare these to the values derived by A13 for a sample of 28 mostly single CTTSs (see Figure 7 from A13). While the NC velocities in our binary stars are similar to the observed averages in the larger sample, we see that the average $\mathrm{BC}$ velocities $\left(\overline{V_{\mathrm{BC}}}=-46 \pm 30 \mathrm{~km} \mathrm{~s}^{-1}\right.$ for DQ Tau, ${ }^{6}$ and $\bar{V}_{\mathrm{BC}}=-17 \pm 20 \mathrm{~km} \mathrm{~s}^{-1}$ for UZ Tau E) are at the low end of what is observed in the larger CTTSs sample $\left(\bar{V}_{\mathrm{BC}}=+40 \pm 50 \mathrm{~km} \mathrm{~s}^{-1}\right)$. For UZ Tau E, the NC line width $\left(\mathrm{FWHM}_{\mathrm{NC}}=50 \pm 10 \mathrm{~km} \mathrm{~s}^{-1}\right)$ is also significantly smaller than the single-star sample average $\left(\mathrm{FWHM}_{\mathrm{NC}}=118 \pm 10 \mathrm{~km} \mathrm{~s}^{-1}\right)$.

In trying to explain the $\mathrm{C}$ IV line shapes, $\mathrm{A} 13$ showed that for accretion rates smaller than $4 \times 10^{-9} M_{\odot} \mathrm{yr}^{-1}$, the average NC contribution to the total luminosity is $\sim 20 \%$, while beyond this limit it is $\sim 40 \%$ on average. This correlation is marginally

\footnotetext{
6 The quoted interval values are the standard deviations, not the errors in the mean.
}

significant. For the binary stars no such effect is detected: the strength of the NC is not correlated with accretion luminosity, $\mathrm{C}$ IV line flux, or accretion rate (as derived from the accretion luminosity). The NC contributes similar amounts of the total C Iv flux in DQ Tau $(30 \% \pm 10 \%)$ and UZ Tau E $(20 \%$ $\pm 10 \%)$.

From the data presented by A13 one can show that the narrow and broad component $\mathrm{C}$ IV luminosities are correlated with each other $(r=0.62, p$-value $=1.5 \%)$, suggesting that both components are powered by the same process, at the same time. As argued in A13, the kinematic evidence for a postshock/pre-shock origin of the NC/BC C Iv line is not clear-cut, and is perhaps being obscured by the curvature of magnetic field lines, inhomogeneities or turbulence in the flow, or the presence of multiple accretion columns. Regardless of this, the correlation is consistent with the $\mathrm{NC}$ and $\mathrm{BC}$ originating in spatially close regions of the system.

Here we compare the C Iv luminosity with the NUV luminosity (Figure 8). This is analogous to Figure 7 but we have replaced the accretion luminosity by the directly observed NUV luminosity. Table 5 lists the correlation coefficients and $p$-values for the quantities in the plot. We use two different 

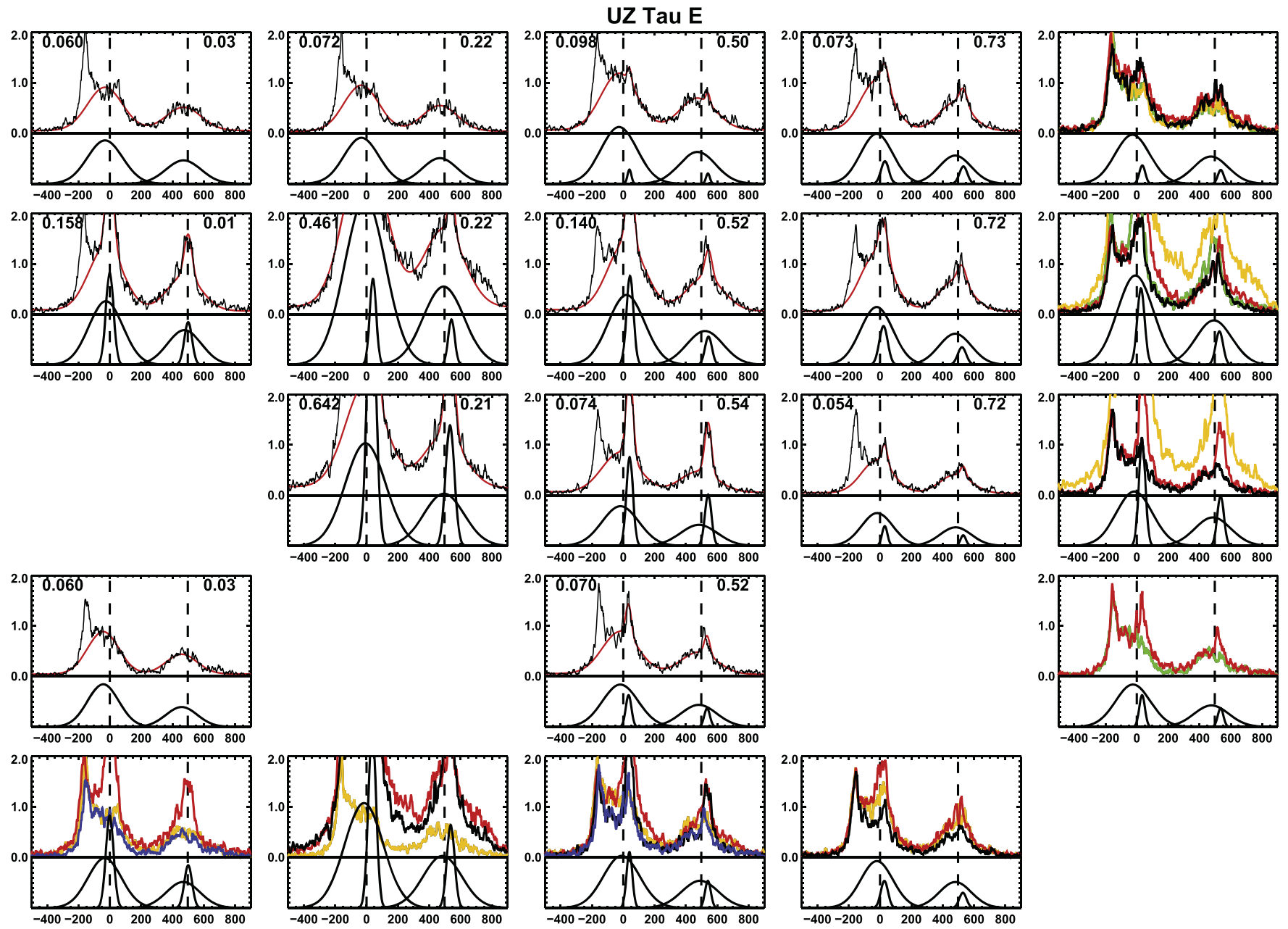

Figure 5. Same as in Figure 4 but for UZ Tau. Empty spaces indicate missing observations. The emission line at $-167 \mathrm{~km} \mathrm{~s}^{-1}$ is the $\mathrm{H}_{2}$ line $R(3) 1-8(1547.3 \AA$ ).
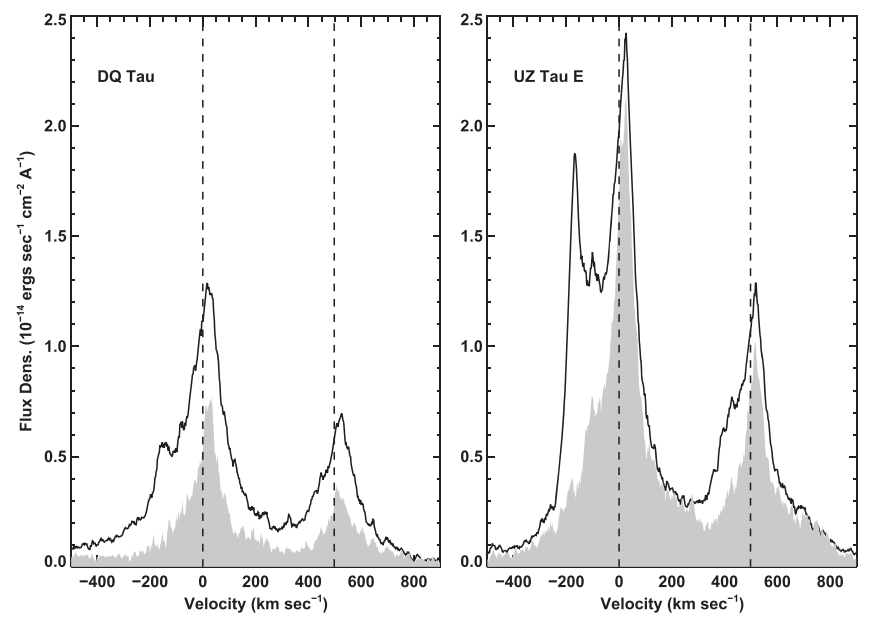

Figure 6. Average (trace) and standard deviation (shaded) of the $\mathrm{C}$ Iv lines shown in Figures 4 and 5. The central narrow cores are significantly more variable than the rest of the lines for both stars.

correlation measures: the Pearson product-moment correlation coefficient $r$ which was used in A13 and the Kendall's $\tau$ nonparametric rank coefficient. The latter does not assume any specific functional form between the quantities being compared.
From Table 5, we conclude that for UZ Tau E and DQ Tau, the correlation between $\mathrm{C}$ IV and NUV luminosity is due to the $\mathrm{BC}$ only, not the NC. As a corollary, $\mathrm{NC}$ and $\mathrm{BC}$ of $\mathrm{C}$ iv are not correlated with each other.

This analysis indicates that unlike the case in single CTTSs, the NCs and BCs in binaries are either powered by a different energy source, have a different spatial origin and/or there is a time delay between the emission of both components. Figure 8 also includes the observed C Iv luminosity in WTTSs, from A13. At least in the case of UZ Tau E, the observed $\mathrm{NC}$ luminosities are within the same range as those observed in WTTSs.

A13 argues that the $\mathrm{NC}$ is being emitted from the post-shock region, because for most CTTSs $V_{\mathrm{NC}}<V_{\mathrm{BC}}$, suggesting that it forms in the decelerating flow closer to the star. Regardless of the specific details of the formation, a naive expectation is that both velocity components should be redshifted, given that the $\mathrm{C}$ IV emission is presumably tracking the end of gas flows falling into the stars. Another is that the velocity of the NC should be a factor of $4 \mathrm{x}$ smaller than the velocity of the $\mathrm{BC}$, the result of formation in the post-shock.

Figure 9 compares the velocities of the narrow and broad line components. The figure also shows the measurements for the larger sample of (mostly) single CTTSs from A13. As is concluded in that work, for the overall sample of CTTS it is generally true that both velocity components are redshifted. 
Table 4

Spectroscopic Measurements

\begin{tabular}{|c|c|c|c|c|c|c|c|c|c|c|c|c|c|c|}
\hline Visit & Target & $\begin{array}{l}\text { Vel. Corr. }^{\mathrm{a}} \\
\left(\mathrm{km} \mathrm{s}^{-1}\right)\end{array}$ & Phase & $\begin{array}{l}L_{\mathrm{NUV}}{ }^{\mathrm{b}} \\
\left(10^{-4} L_{\odot}\right)\end{array}$ & $\begin{array}{l}L_{\mathrm{Acc}}{ }^{\mathrm{c}} \\
\left(L_{\odot}\right)\end{array}$ & C Iv Flux ${ }^{\mathrm{d}}$ & $\begin{array}{l}A_{\mathrm{NC}_{1}}{ }^{\mathrm{e}} \\
(\mathrm{FU})^{\mathrm{f}}\end{array}$ & $\begin{array}{l}A_{\mathrm{NC}_{2}} \\
(\mathrm{FU})^{\mathrm{f}}\end{array}$ & $\begin{array}{l}V_{\mathrm{NC}} \\
\left(\mathrm{km} \mathrm{s}^{-1}\right)\end{array}$ & $\begin{array}{l}\sigma_{\mathrm{NC}} \\
\left(\mathrm{km} \mathrm{s}^{-1}\right)\end{array}$ & $\begin{array}{l}A_{\mathrm{BC}_{1}} \\
(\mathrm{FU})^{\mathrm{f}}\end{array}$ & $\begin{array}{l}A_{\mathrm{BC}_{2}} \\
(\mathrm{FU})^{\mathrm{f}}\end{array}$ & $\begin{array}{l}V_{\mathrm{BC}} \\
\left(\mathrm{km} \mathrm{s}^{-1}\right)\end{array}$ & $\begin{array}{l}\sigma_{\mathrm{BC}} \\
\left(\mathrm{km} \mathrm{s}^{-1}\right)\end{array}$ \\
\hline & Ave. $^{g}$ & $-0.41 \pm 0.9$ & & $5.2 \pm 8.3$ & $0.13 \pm 0.2$ & $2.16 \pm 0.7$ & $0.80 \pm 0.5$ & $0.48 \pm 0.3$ & $21 \pm 10$ & $51 \pm 20$ & $0.44 \pm 0.2$ & $0.21 \pm 0.1$ & $-46 \pm 30$ & $176 \pm 50$ \\
\hline 13 & DQ Tau & -2.84 & 0.00 & $\ldots$ & $\ldots$ & $2.90 \pm 0.05$ & $\begin{array}{l}0.71 \\
\quad \pm 0.03\end{array}$ & $\begin{array}{l}0.42 \\
\quad \pm 0.02\end{array}$ & $28.3 \pm 0.6$ & $81 \pm 1$ & $\begin{array}{l}0.39 \\
\quad \pm 0.03\end{array}$ & $\begin{array}{l}0.21 \\
\quad \pm 0.02\end{array}$ & $-26 \pm 2$ & $221 \pm 4$ \\
\hline 14 & DQ Tau & -0.04 & 0.24 & $1.52 \pm 0.09$ & $\begin{array}{l}0.03 \\
\quad \pm 0.02\end{array}$ & $1.05 \pm 0.03$ & $\begin{array}{l}0.34 \\
\quad \pm 0.03\end{array}$ & $\begin{array}{l}0.28 \\
\quad \pm 0.01\end{array}$ & $18.3 \pm 0.7$ & $36 \pm 1$ & $\begin{array}{l}0.21 \\
\quad \pm 0.03\end{array}$ & $\begin{array}{l}0.12 \\
\quad \pm 0.01\end{array}$ & $-39 \pm 1$ & $150 \pm 3$ \\
\hline 15 & DQTau & -0.83 & 0.54 & $6.92 \pm 0.12$ & $\begin{array}{l}0.17 \\
\quad \pm 0.06\end{array}$ & $2.77 \pm 0.05$ & $\begin{array}{l}1.82 \\
\quad \pm 0.04\end{array}$ & $\begin{array}{l}0.78 \\
\quad \pm 0.02\end{array}$ & $36.7 \pm 0.3$ & $23.3 \pm 0.2$ & $\begin{array}{l}0.83 \\
\quad \pm 0.04\end{array}$ & $\begin{array}{l}0.37 \\
\quad \pm 0.02\end{array}$ & $-5.7 \pm 0.2$ & $128 \pm 1$ \\
\hline 16 & DQ Tau & -0.27 & 0.7 & $\begin{array}{l}29.06 \\
\quad \pm 0.20\end{array}$ & $\begin{array}{l}0.79 \\
\quad \pm 0.20\end{array}$ & $3.55 \pm 0.06$ & $\begin{array}{l}1.56 \\
\quad \pm 0.06\end{array}$ & $\begin{array}{l}1.23 \\
\quad \pm 0.03\end{array}$ & $16.3 \pm 0.3$ & $27.7 \pm 0.5$ & $\begin{array}{l}0.79 \\
\quad \pm 0.06\end{array}$ & $\begin{array}{l}0.52 \\
\quad \pm 0.03\end{array}$ & $-12.8 \pm 0.2$ & $117 \pm 1$ \\
\hline 17 & DQ Tau & 0.42 & 0.03 & $1.89 \pm 0.09$ & $\begin{array}{l}0.04 \\
\quad \pm 0.02\end{array}$ & $2.54 \pm 0.04$ & $\begin{array}{l}0.58 \\
\quad \pm 0.03\end{array}$ & $\begin{array}{l}0.74 \\
\quad \pm 0.02\end{array}$ & $39.8 \pm 1$ & $44 \pm 1$ & $\begin{array}{l}0.34 \\
\quad \pm 0.03\end{array}$ & $\begin{array}{l}0.33 \\
\quad \pm 0.02\end{array}$ & $-16.1 \pm 0.8$ & $141 \pm 1$ \\
\hline 18 & DQ Tau & 0.76 & 0.24 & $1.28 \pm 0.09$ & $\begin{array}{l}0.03 \\
\quad \pm 0.01\end{array}$ & $1.59 \pm 0.04$ & $\begin{array}{l}0.47 \\
\quad \pm 0.03\end{array}$ & $\begin{array}{l}0.33 \\
\quad \pm 0.02\end{array}$ & $10.0 \pm 0.7$ & $58 \pm 2$ & $\begin{array}{l}0.24 \\
\quad \pm 0.03\end{array}$ & $\begin{array}{l}0.15 \\
\quad \pm 0.02\end{array}$ & $-50 \pm 1$ & $169 \pm 3$ \\
\hline 19 & DQ Tau & -0.03 & 0.51 & $3.16 \pm 0.10$ & $\begin{array}{l}0.07 \\
\quad \pm 0.03\end{array}$ & $2.29 \pm 0.04$ & $\begin{array}{l}1.04 \\
\quad \pm 0.03\end{array}$ & $\begin{array}{l}0.38 \\
\quad \pm 0.02\end{array}$ & $3.2 \pm 0.1$ & $48.4 \pm 0.5$ & $\begin{array}{l}0.58 \\
\quad \pm 0.03\end{array}$ & $\begin{array}{l}0.18 \\
\quad \pm 0.01\end{array}$ & $-51 \pm 2$ & $176 \pm 3$ \\
\hline 20 & DQ Tau & -0.8 & 0.7 & $4.00 \pm 0.11$ & $\begin{array}{l}0.09 \\
\quad \pm 0.04\end{array}$ & $1.92 \pm 0.04$ & $\begin{array}{l}0.46 \\
\quad \pm 0.03\end{array}$ & $\begin{array}{l}0.42 \\
\quad \pm 0.02\end{array}$ & $11.1 \pm 0.7$ & $56 \pm 1$ & $\begin{array}{l}0.36 \\
\quad \pm 0.03\end{array}$ & $\begin{array}{l}0.15 \\
\quad \pm 0.02\end{array}$ & $-38 \pm 1$ & $179 \pm 3$ \\
\hline 21 & DQ Tau & -0.19 & 0.04 & $5.03 \pm 0.11$ & $\begin{array}{l}0.12 \\
\quad \pm 0.05\end{array}$ & $2.39 \pm 0.05$ & $\begin{array}{l}1.24 \\
\quad \pm 0.02\end{array}$ & $\begin{array}{l}0.25 \\
\quad \pm 0.01\end{array}$ & $28.4 \pm 0.3$ & $45.9 \pm 0.4$ & $\begin{array}{l}0.67 \\
\quad \pm 0.02\end{array}$ & $\begin{array}{l}0.06 \\
\quad \pm 0.00\end{array}$ & $-54.4 \pm 0.8$ & $305 \pm 3$ \\
\hline 22 & DQ Tau & -0.28 & 0.21 & $1.22 \pm 0.09$ & $\begin{array}{l}0.03 \\
\quad \pm 0.01\end{array}$ & $1.81 \pm 0.04$ & $\begin{array}{l}0.43 \\
\quad \pm 0.03\end{array}$ & $\begin{array}{l}0.45 \\
\quad \pm 0.02\end{array}$ & $14.1 \pm 0.6$ & $61 \pm 1$ & $\begin{array}{l}0.32 \\
\quad \pm 0.03\end{array}$ & $\begin{array}{l}0.16 \\
\quad \pm 0.02\end{array}$ & $-81 \pm 1$ & $157 \pm 2$ \\
\hline 23 & DQ Tau & -0.68 & 0.52 & $0.77 \pm 0.09$ & $\begin{array}{l}0.02 \\
\quad \pm 0.01\end{array}$ & $1.11 \pm 0.03$ & $\begin{array}{l}0.28 \\
\quad \pm 0.02\end{array}$ & $\begin{array}{l}0.29 \\
\quad \pm 0.01\end{array}$ & $32.2 \pm 1$ & $39 \pm 2$ & $\begin{array}{l}0.19 \\
\quad \pm 0.02\end{array}$ & $\begin{array}{l}0.13 \\
\quad \pm 0.01\end{array}$ & $-85 \pm 2$ & $157 \pm 3$ \\
\hline \multirow[t]{2}{*}{24} & DQ Tau & 0.03 & 0.73 & $2.30 \pm 0.10$ & $\begin{array}{l}0.05 \\
\quad \pm 0.02\end{array}$ & $1.94 \pm 0.04$ & $\begin{array}{l}0.62 \\
\quad \pm 0.02\end{array}$ & $\begin{array}{l}0.22 \\
\quad \pm 0.02\end{array}$ & $19.3 \pm 1$ & $89 \pm 2$ & $\begin{array}{l}0.31 \\
\quad \pm 0.02\end{array}$ & $\begin{array}{l}0.10 \\
\quad \pm 0.02\end{array}$ & $-88 \pm 4$ & $209 \pm 7$ \\
\hline & Ave. & $-2.09 \pm 0.9$ & & $13.7 \pm 14.7$ & $\begin{array}{l}0.17 \\
\quad \pm 0.19\end{array}$ & $3.4 \pm 2$ & $1.5 \pm 1.4$ & $1.2 \pm 0.7$ & $32 \pm 10$ & $21.5 \pm 5$ & $0.70 \pm 0.7$ & $0.64 \pm 0.3$ & $-17 \pm 20$ & $112 \pm 10$ \\
\hline 1 & UZ Tau E & -3.27 & 0.03 & $5.59 \pm 0.12$ & $\begin{array}{l}0.06 \\
\quad \pm 0.03\end{array}$ & $1.93 \pm 0.04$ & $\ldots$ & $\begin{array}{l}0.86 \\
\quad \pm 0.02\end{array}$ & $\ldots$ & $\cdots$ & $\ldots$ & $\begin{array}{l}0.46 \\
\quad \pm 0.01\end{array}$ & $-30.3 \pm 0.5$ & $112 \pm 1$ \\
\hline 2 & UZ Tau E & -2.29 & 0.22 & $6.64 \pm 0.12$ & $\begin{array}{l}0.08 \\
\quad \pm 0.03\end{array}$ & $2.07 \pm 0.04$ & $\cdots$ & $\begin{array}{l}0.91 \\
\quad \pm 0.02\end{array}$ & $\ldots$ & $\cdots$ & $\cdots$ & $\begin{array}{l}0.51 \\
\quad \pm 0.01\end{array}$ & $-32.8 \pm 0.5$ & $111.1 \pm 1.0$ \\
\hline 3 & UZ Tau E & -1.06 & 0.5 & $8.84 \pm 0.12$ & $\begin{array}{l}0.10 \\
\quad \pm 0.04\end{array}$ & $2.88 \pm 0.05$ & $\begin{array}{l}0.28 \\
\quad \pm 0.06\end{array}$ & $\begin{array}{l}1.12 \\
\quad \pm 0.02\end{array}$ & $39.4 \pm 2$ & $12 \pm 2$ & $\begin{array}{l}0.20 \\
\quad \pm 0.06\end{array}$ & $\begin{array}{l}0.63 \\
\quad \pm 0.02\end{array}$ & $-27.0 \pm 0.4$ & $116 \pm 1$ \\
\hline 4 & UZ Tau E & -0.24 & 0.73 & $6.78 \pm 0.11$ & $\begin{array}{l}0.08 \\
\quad \pm 0.04\end{array}$ & $2.32 \pm 0.05$ & $\begin{array}{l}0.45 \\
\quad \pm 0.05\end{array}$ & $\begin{array}{l}0.99 \\
\quad \pm 0.02\end{array}$ & $31.4 \pm 1$ & $25 \pm 1$ & $\begin{array}{l}0.34 \\
\quad \pm 0.04\end{array}$ & $\begin{array}{l}0.56 \\
\quad \pm 0.02\end{array}$ & $-21.4 \pm 0.4$ & $102 \pm 1$ \\
\hline 5 & UZ Tau E & -3.43 & 0.01 & $\begin{array}{l}13.73 \\
\quad \pm 0.15\end{array}$ & $\begin{array}{l}0.17 \\
\quad \pm 0.06\end{array}$ & $3.63 \pm 0.06$ & $\begin{array}{l}1.83 \\
\quad \pm 0.05\end{array}$ & $\begin{array}{l}1.26 \\
\quad \pm 0.03\end{array}$ & $-0.4 \pm 0.2$ & $24.6 \pm 0.3$ & $\begin{array}{l}0.85 \\
\quad \pm 0.05\end{array}$ & $\begin{array}{l}0.69 \\
\quad \pm 0.03\end{array}$ & $-24.7 \pm 0.4$ & $108.3 \pm 0.9$ \\
\hline 6 & UZ Tau E & -2.8 & 0.22 & $\begin{array}{l}36.45 \\
\quad \pm 0.22\end{array}$ & $\begin{array}{l}0.49 \\
\quad \pm 0.14\end{array}$ & $8.69 \pm 0.08$ & $\begin{array}{l}1.63 \\
\quad \pm 0.08\end{array}$ & $\begin{array}{l}3.17 \\
\quad \pm 0.03\end{array}$ & $41.2 \pm 0.5$ & $21.0 \pm 0.5$ & $\begin{array}{l}0.90 \\
\quad \pm 0.08\end{array}$ & $\begin{array}{l}1.58 \\
\quad \pm 0.03\end{array}$ & $-8.9 \pm 0.2$ & $129.7 \pm 0.6$ \\
\hline 7 & UZ Tau E & -3.11 & 0.52 & $\begin{array}{l}12.28 \\
\quad \pm 0.14\end{array}$ & $\begin{array}{l}0.15 \\
\quad \pm 0.06\end{array}$ & $3.67 \pm 0.06$ & $\begin{array}{l}1.76 \\
\quad \pm 0.06\end{array}$ & $\begin{array}{l}1.38 \\
\quad \pm 0.03\end{array}$ & $43.0 \pm 0.4$ & $22.2 \pm 0.5$ & $\begin{array}{l}0.56 \\
\quad \pm 0.06\end{array}$ & $\begin{array}{l}0.67 \\
\quad \pm 0.03\end{array}$ & $20.5 \pm 0.3$ & $115.9 \pm 0.8$ \\
\hline 8 & UZ Tau E & -1.66 & 0.72 & $\ldots$ & $\ldots$ & $2.74 \pm 0.05$ & $\begin{array}{l}0.77 \\
\quad \pm 0.05\end{array}$ & $\begin{array}{l}1.14 \\
\quad \pm 0.03\end{array}$ & $22.2 \pm 0.9$ & $26.9 \pm 0.9$ & $\begin{array}{l}0.35 \\
\quad \pm 0.05\end{array}$ & $\begin{array}{l}0.62 \\
\quad \pm 0.02\end{array}$ & $-21.3 \pm 0.4$ & $103 \pm 1$ \\
\hline 10 & UZ Tau E & -1.96 & 0.21 & $\begin{array}{l}49.52 \\
\quad \pm 0.25\end{array}$ & $\begin{array}{l}0.68 \\
\quad \pm 0.18\end{array}$ & $8.04 \pm 0.08$ & $\begin{array}{l}5.39 \\
\quad \pm 0.07\end{array}$ & $\begin{array}{l}2.03 \\
\quad \pm 0.03\end{array}$ & $31.8 \pm 0.2$ & $28.3 \pm 0.2$ & $\begin{array}{l}2.39 \\
\quad \pm 0.06\end{array}$ & $\begin{array}{l}1.03 \\
\quad \pm 0.03\end{array}$ & $-6.9 \pm 0.4$ & $129 \pm 1$ \\
\hline 11 & UZ Tau E & -2.68 & 0.54 & $6.86 \pm 0.13$ & & $2.56 \pm 0.05$ & & & $40.9 \pm 0.4$ & $21.1 \pm 0.2$ & & & $-18.0 \pm 0.5$ & $118 \pm 1$ \\
\hline
\end{tabular}


Table 4

(Continued)

\begin{tabular}{|c|c|c|c|c|c|c|c|c|c|c|c|c|c|c|}
\hline Visit & Target & $\begin{array}{c}\text { Vel. Corr. }^{\mathrm{a}} \\
\left(\mathrm{km} \mathrm{s}^{-1}\right)\end{array}$ & Phase & $\begin{array}{l}L_{\mathrm{NUV}}{ }^{\mathrm{b}} \\
\left(10^{-4} L_{\odot}\right)\end{array}$ & $\begin{array}{l}L_{\mathrm{Acc}}{ }^{\mathrm{c}} \\
\left(L_{\odot}\right)\end{array}$ & C Iv Flux ${ }^{\mathrm{d}}$ & $\begin{array}{l}A_{\mathrm{NC}_{1}}{ }^{\mathrm{e}} \\
(\mathrm{FU})^{\mathrm{f}}\end{array}$ & $\begin{array}{l}A_{\mathrm{NC}_{2}} \\
(\mathrm{FU})^{\mathrm{f}}\end{array}$ & $\begin{array}{l}V_{\mathrm{NC}} \\
\left(\mathrm{km} \mathrm{s}^{-1}\right)\end{array}$ & $\begin{array}{l}\sigma_{\mathrm{NC}} \\
\left(\mathrm{km} \mathrm{s}^{-1}\right)\end{array}$ & $\begin{array}{l}A_{\mathrm{BC}_{1}} \\
(\mathrm{FU})^{\mathrm{f}}\end{array}$ & $\begin{array}{l}A_{\mathrm{BC}_{2}} \\
(\mathrm{FU})^{\mathrm{f}}\end{array}$ & $\begin{array}{l}V_{\mathrm{BC}} \\
\left(\mathrm{km} \mathrm{s}^{-1}\right)\end{array}$ & $\begin{array}{l}\sigma_{\mathrm{BC}} \\
\left(\mathrm{km} \mathrm{s}^{-1}\right)\end{array}$ \\
\hline & & & & & $\begin{array}{l}0.08 \\
\quad \pm 0.04\end{array}$ & & $\begin{array}{l}1.76 \\
\quad \pm 0.05\end{array}$ & $\begin{array}{l}0.79 \\
\quad \pm 0.02\end{array}$ & & & $\begin{array}{l}1.02 \\
\quad \pm 0.05\end{array}$ & $\begin{array}{l}0.42 \\
\quad \pm 0.02\end{array}$ & & \\
\hline 12 & UZ Tau E & -1.52 & 0.72 & $5.14 \pm 0.11$ & $\begin{array}{l}0.06 \\
\quad \pm 0.03\end{array}$ & $1.40 \pm 0.04$ & $\begin{array}{l}0.39 \\
\quad \pm 0.05\end{array}$ & $\begin{array}{l}0.65 \\
\quad \pm 0.02\end{array}$ & $30.2 \pm 1$ & $17.1 \pm 0.8$ & $\begin{array}{l}0.21 \\
\quad \pm 0.04\end{array}$ & $\begin{array}{l}0.37 \\
\quad \pm 0.02\end{array}$ & $-18.2 \pm 0.5$ & $100 \pm 1$ \\
\hline 26 & UZ Tau E & -1.82 & 0.03 & $5.60 \pm 0.11$ & $\begin{array}{l}0.06 \\
\quad \pm 0.03\end{array}$ & $1.51 \pm 0.04$ & $\ldots$ & $\begin{array}{l}0.84 \\
\quad \pm 0.02\end{array}$ & $\ldots$ & $\ldots$ & $\ldots$ & $\begin{array}{l}0.39 \\
\quad \pm 0.02\end{array}$ & $-42.1 \pm 0.6$ & $100 \pm 1$ \\
\hline 25 & UZ Tau E & -1.33 & 0.52 & $6.52 \pm 0.12$ & $\begin{array}{l}0.07 \\
\quad \pm 0.03\end{array}$ & $2.15 \pm 0.04$ & $\begin{array}{l}0.63 \\
\quad \pm 0.05\end{array}$ & $\begin{array}{l}0.84 \\
\quad \pm 0.02\end{array}$ & $34.0 \pm 0.8$ & $18.0 \pm 0.5$ & $\begin{array}{l}0.37 \\
\quad \pm 0.04\end{array}$ & $\begin{array}{l}0.44 \\
\quad \pm 0.02\end{array}$ & $-18.7 \pm 0.5$ & $115 \pm 1$ \\
\hline
\end{tabular}

Notes.

${ }^{\text {a }}$ Velocity correction due to pointing errors. To be applied to measured velocities.

${ }^{\mathrm{b}}$ Integrated NUV luminosity, not corrected for extinction.

${ }^{c}$ Accretion Luminosity.

${ }^{\mathrm{d}}$ Measured $\mathrm{C}$ iv flux. Not corrected for extinction. $10^{-14} \mathrm{erg} \mathrm{s}^{-1} \mathrm{~cm}^{-2}$.

${ }^{\mathrm{e}}$ Gaussian fits to the $\mathrm{C}$ iv lines: $F=A_{\mathrm{NC}_{1}} \exp \left(-\left(v-V_{\mathrm{NC}}\right)^{2 / 2 \sigma_{\mathrm{NC}}^{2}}\right)+A_{\mathrm{BC}_{1}} \exp \left(-\left(v-V_{\mathrm{BC}}\right)^{2 / 2 \sigma_{\mathrm{BC}}^{2}}\right)+A_{\mathrm{NC}_{2}} \exp \left(-\left(v-\left(500.96+V_{\mathrm{NC}}\right)\right)^{2 / 2 \sigma_{\mathrm{NC}}^{2}}\right)+A_{\mathrm{BC}} \exp \left(-\left(v-\left(500.96+V_{\mathrm{BC}}\right)^{2} / 2 \sigma_{\mathrm{BC}}^{2}\right)\right.$.

${ }^{f}$ FU: Flux units: $10^{-14} \mathrm{erg} \mathrm{s}^{-1} \mathrm{~cm}^{-2} \AA^{-1}$.

$\mathrm{g}$ Average quantities and rms of the measured values for DQ Tau.

${ }^{\mathrm{h}}$ Average quantities and rms of the measured values for UZ Tau E. 

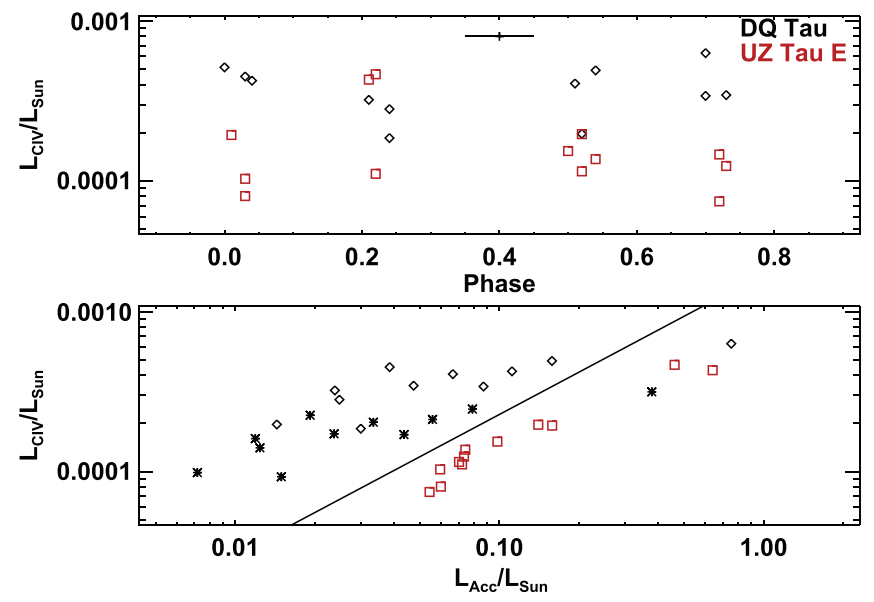

Figure 7. Top: $\mathrm{C}$ iv line luminosity vs. phase. The average error in the phase is indicated by the horizontal bar. For the number of observations presented here we do not detect any correlation between these two quantities. Bottom: $\mathrm{C}$ IV line luminosity vs. accretion luminosity. The solid line is the relationship derived by Yang et al. (2012). The asterisk symbols correspond to DQ Tau, assuming that each star contributes equally to the $C_{\text {IV }}$ and accretion luminosities. C IV and $L_{\mathrm{Acc}}$ errors: $\pm 1 \%$ to $3 \%$.

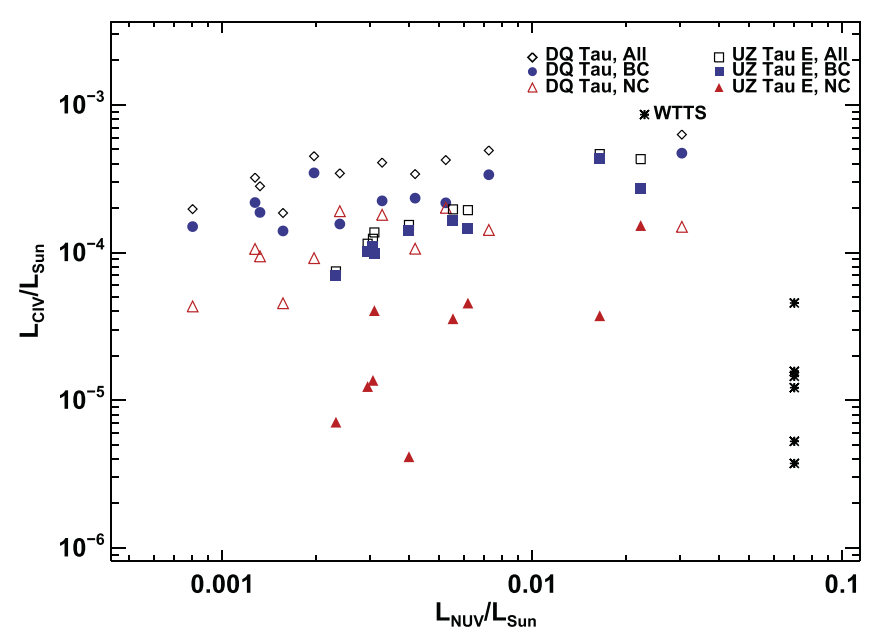

Figure 8. C Iv luminosity is correlated with the measured NUV luminosity, which tracks the accretion rate. However, this correlation is due to the broad components of the line only. $L_{\mathrm{Cr}, \mathrm{NC}}$ and $L_{\mathrm{NUV}}$ are not correlated. The random measurement errors (given in Table 4) are 1\%-3\% for the $\mathrm{BC}$ luminosities, $1 \%-20 \%$ for the NC luminosities, $1 \%-3 \%$ for $L_{\mathrm{NUV}}$, and $1 \%-10 \%$ for $L_{\mathrm{Crv}}$. For comparison, WTTSs C iv luminosities are also shown, at an arbitrary abcisa value.

This is not the case for DQ Tau and UZ Tau E. For both we see negative $\mathrm{BC}$ velocities for most observations, and in no case is $V_{\mathrm{NC}} \lesssim 4 V_{\mathrm{BC}}$

\subsection{Accretion to a Single Star?}

We do not observe any line splitting in either of the two systems at any phase. For DQ Tau this is not unexpected given that $\overline{\mathrm{FWHM}}_{\mathrm{NC}}=120 \mathrm{~km} \mathrm{~s}^{-1}$ but the largest sampled velocity difference between primary and secondary is $\sim 40 \mathrm{~km} \mathrm{~s}^{-1}$ (at phase 0.0). Consistent with this, detailed simulations of our observing setup show that even if both stars in the system were contributing the same amount of flux to the observed line we would not be able to detect line splitting. For UZ Tau E, the width of the $\mathrm{NC}\left(\overline{\mathrm{FWHM}}_{\mathrm{NC}}=50 \mathrm{~km} \mathrm{~s}^{-1}\right)$ is significantly smaller that the single CTTS average $\left(\overline{\mathrm{FWHM}}_{\mathrm{NC}}=118\right.$
Table 5

Correlations between the $L_{\mathrm{NUV}}$ and $L_{\mathrm{CIV}}$

\begin{tabular}{|c|c|c|c|c|c|}
\hline Quantities & $r^{\mathrm{a}}$ & $p$-val. $^{\mathrm{b}}$ & $\tau^{\mathrm{c}}$ & $p$-val. ${ }^{\mathrm{d}}$ & Corr.? \\
\hline \multicolumn{6}{|c|}{ DQ Tau } \\
\hline$L_{\mathrm{NUV}}, L_{\mathrm{CIV}}$ & 0.77 & 0.005 & 0.65 & 0.005 & $\mathrm{Y}$ \\
\hline$L_{\mathrm{NUV}}, L_{\mathrm{BC}}$ & 0.81 & 0.003 & 0.79 & 0.004 & $\mathrm{Y}$ \\
\hline$L_{\mathrm{NUV}}, L_{\mathrm{NC}}$ & 0.29 & 0.4 & 0.49 & 0.04 & $\mathrm{~N}$ \\
\hline$L_{\mathrm{BC}}, L_{\mathrm{NC}}$ & 0.24 & 0.5 & 0.20 & 0.39 & $\mathrm{~N}$ \\
\hline \multicolumn{6}{|c|}{ UZ Tau E } \\
\hline$L_{\mathrm{NUV}}, L_{\mathrm{CIV}}$ & 0.96 & $210^{-4}$ & 0.89 & 0.001 & $\mathrm{Y}$ \\
\hline$L_{\mathrm{NUV}}, L_{\mathrm{BC}}$ & 0.84 & 0.005 & 0.78 & 0.004 & $\mathrm{Y}$ \\
\hline$L_{\mathrm{NUV}}, L_{\mathrm{NC}}$ & 0.84 & 0.004 & 0.61 & 0.03 & $\mathrm{~N}$ \\
\hline$L_{\mathrm{BC}}, L_{\mathrm{NC}}$ & 0.44 & 0.2 & 0.39 & 0.14 & $\mathrm{~N}$ \\
\hline
\end{tabular}

Notes.

a Pearson's $r$ product moment correlation coefficient.

${ }^{\mathrm{b}} p$-value for $r$.

${ }^{c}$ Kendall's $\tau$ rank coefficient.

d $p$-value for $\tau$.

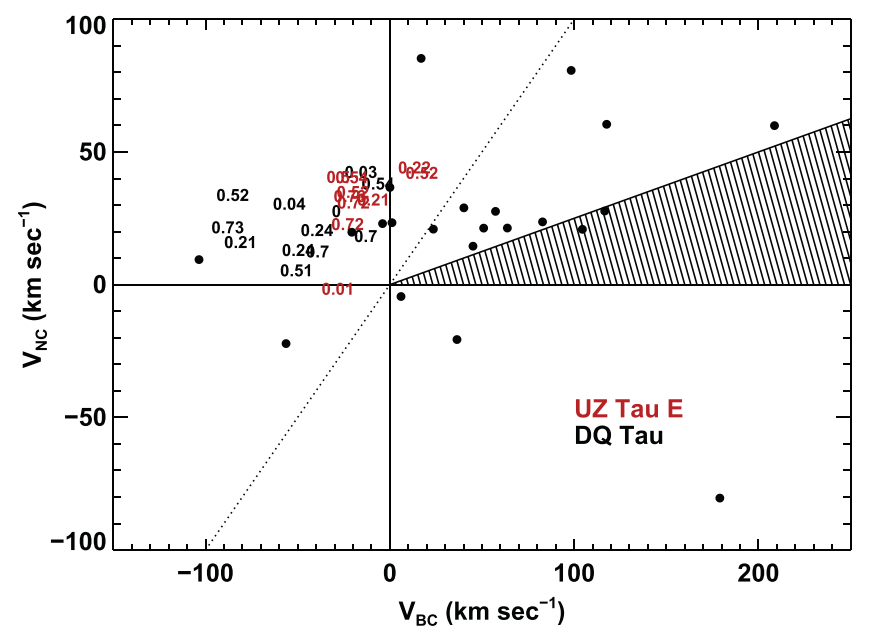

Figure 9. Velocity of the narrow Gaussian component vs. the broad Gaussian component for $\mathrm{C}$ Iv. Each observation is marked according to phase. The filledcircle symbols are CTTSs from the A13 sample. The hatched region marks $V_{\mathrm{NC}}=V_{\mathrm{BC}} / 4$, where the points should reside if their origin were purely magnetospheric. The dotted line is the identity relationship.

$\mathrm{km} \mathrm{s}^{-1}$ ). The maximum velocity difference between primary and secondary is $\sim 60 \mathrm{~km} \mathrm{~s}^{-1}$ at phase 0.2 . We should be able to detect line splitting if the contributions from both stars were comparable.

In addition, we do not find any correlation between line width and the separation between primary and secondary or their velocity difference, for either of the systems.

If the observed emission is due to only one of the stars, the $\mathrm{C}$ IV line should reflect the kinematics of its source. Figure 10 compares the $\mathrm{NC} \mathrm{C}$ iv velocity to the primary velocity in $\mathrm{DQ}$ Tau and UZ Tau E. The velocity of the C Iv line centroid reflects not only the kinematics of the stars as they orbit around their center-of-mass, but also the intrinsic velocity of the accretion flow. In the A13 sample, the average velocity centroid of the NC in CTTSs is $+26.6 \mathrm{~km} \mathrm{~s}^{-1}$, and in Figure 10 we have arbitrarily subtracted $+26.6 \mathrm{~km} \mathrm{~s}^{-1}$ from both stars' velocities.

No correlation between $\mathrm{NC}$ velocity and primary velocity is observed for DQ Tau, suggesting that the dominant emission 

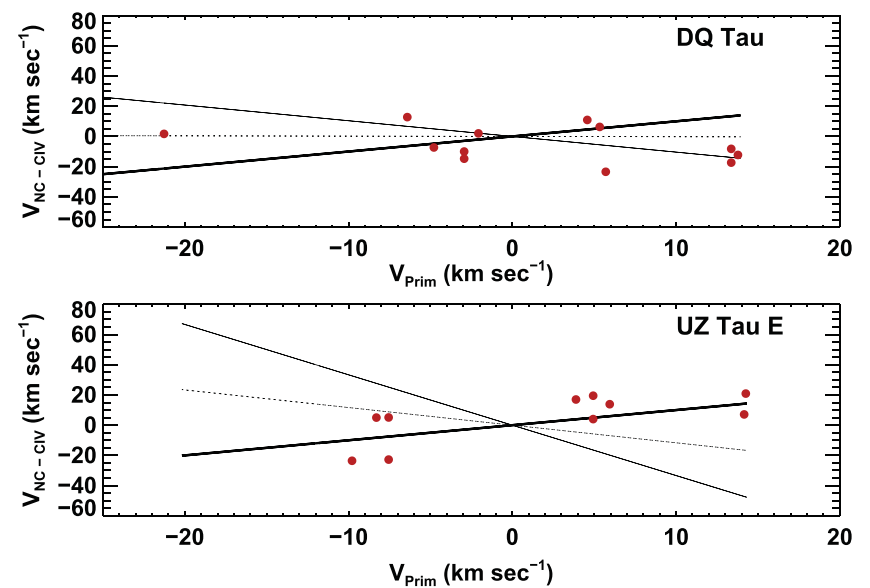

Figure 10. Velocity of the NC of C IV vs. the velocity of the primary. The data are given by the red filled-circles. The dotted line is the expected correlation if both stars contribute equally to the $\mathrm{C}$ IV line. The positive (negative) slope solid line is the expected correlation if only the primary (secondary) contributes to the $\mathrm{C}$ Iv line. The $\mathrm{NC}$ of the $\mathrm{C}$ Iv line for $\mathrm{UZ}$ Tau $\mathrm{E}$ follows the primary $(r=0.7, p=2.5 \%)$.

region changes from orbit to orbit or that both system members contribute equally to the formation of the line.

For UZ Tau E, we find that $V_{\mathrm{NC}}$ is correlated with the primary velocity $(r=0.70, p$-value $=2.5 \%)$. There is enough scatter in the points such that the observations do not rule out that some of the $\mathrm{NC}$ emission is originating in the secondary, but this is not the dominant component of the emission.

There are, however, additional effects that complicate the interpretation of the observed C IV centroid velocities, such as astrometric shifts, intrinsic velocity changes, and stellar rotation.

In principle, the motion of the binary members around their center of mass may result in a change in the position of the observed light peak, causing an apparent change in line velocity. However, the largest apparent separation between the members of the binaries is $\sim 40 R_{\odot}$, corresponding to $\sim 1$ milliarcsecond and such shift is not detectable with the current calibration (Section 3).

The observed velocities of the $C$ IV profiles may change due to increased accretion rates that result in loading of different magnetic lines and therefore different free-fall velocities. Figure 10 from A13 shows examples of $\mathrm{C}$ IV line profile changes for a handful of stars. For RU Lup we see NC velocity changes of almost $30 \mathrm{~km} \mathrm{~s}^{-1}$ between different epoch observations taken with $H S T /$ STIS and HST/COS. Others such as DF Tau and BP Tau show smaller NC velocity changes, consistent with the systematic uncertainty in the $H S T /$ GHRS wavelength scale.

The effect of stellar rotation in the velocity centroid of the $\mathrm{C}$ IV lines is uncertain. Even assuming that the rotation axis of the star is the same as the rotation axis of the binary, we do not know where the accretion column reaches the stellar surface. Zeeman-Doppler imaging studies (see Gregory et al. 2012 and references therein) indicate that accretion occurs close to the magnetic poles, but the angle between the poles and the rotation axis for these stars is unknown. Observed line velocities may be shifted by an amount as large as the measured rotational velocity ( $v \sin i=10 \pm 2 \mathrm{~km} \mathrm{~s}^{-1}$ for DQ Tau, $v \sin i=16 \mathrm{~km} \mathrm{~s}^{-1}$ for UZ Tau E).
It is likely that those effects contribute to the scatter in the observations presented in Figure 10 although neither of them is important enough to destroy the correlation between $V_{\mathrm{NC}}$ and $V_{\text {Prim }}$ for UZ Tau E.

\section{DISCUSSION AND CONCLUSIONS}

Our goal in this work has been to understand the accretion process in binary systems, using the FUV C IV line, the NUV continuum, and ground-based photometric observations as observational proxies. In single CTTSs the $\mathrm{C}$ IV line tracks the footpoint of magnetically funneled accretion flows. The NUV continuum is the result of the energy reprocessing at the bottom of the accretion stream.

We obtained $H S T / C O S$ spectra $(R \sim 20,000$ and $R \sim 3000)$ of DQ Tau and UZ Tau E, young spectroscopic binaries surrounded by circumbinary disks. Each system was observed over 3 or 4 consecutive orbits, at phase points $\sim 0,0.2,0.5$, and 0.7. In addition, we obtained simultaneous optical and near-IR data. Here we focus on the COS data plus the ground-based U-band measurements.

These binaries provide us with different conditions in which to study accretion: DQ Tau is an equal mass binary with high eccentricity, while UZ Tau $\mathrm{E}$ is an unequal mass $\left(M_{2} /\right.$ $M_{1}=0.3$ ) binary with lower eccentricity. For DQ Tau, the separation between the stars range from 13 to $45 R_{\odot}$ while for UZ Tau E it ranges from 24 to $48 R_{\odot}$. Given the characteristics of the systems, our analysis indicates that no stable, long-lived circumstellar disks exists around the members of DQ Tau nor around the secondary of UZ Tau E. There may be a small circumstellar disk around the UZ Tau E primary.

A key prediction of the dynamical models of accretion in binary systems is that the accretion rate is periodic with the orbital period. We use simultaneous U-band measurements to convert the NUV continuum luminosity into a U-band luminosity, and then into accretion luminosity, via the relationship derived by Gullbring et al. (1998). To track the behavior of accretion with phase we also use archival U-band measurements (Herbst et al. 1994), as well as our own groundbased U-band observations.

For DQ Tau we do not observe any correlation between accretion luminosity measured by the NUV continuum and orbital phase. Archival U-band photometry shows frequent enhancement of the accretion luminosity at periastron, similar to what has been reported before (Mathieu et al. 1997). This enhancement does not occur at every periastron passage, which is different from the AL96 model predictions. We find one instance of very large $(\sim 10 \times)$ accretion luminosity far from periastron. This behavior suggests that gas is stored and accumulated in the system throughout the orbit and may accrete stochastically.

UZ Tau E is a less ideal system to test the relationship between accretion and phase, as models predict very broad accretion maxima. Our observations are consistent with previous ones (Jensen et al. 2007), but again showing examples of very large occasional accretion luminosities at the "wrong" phase.

We do not detect a correlation between $\mathrm{C}$ IV line flux and phase. The C IV line flux is correlated with NUV and accretion luminosity, as in the general sample of CTTSs (Yang et al. 2012).

In both targets the appearance of the $\mathrm{C}$ IV line is similar to that of single CTTSs: a $50-100 \mathrm{~km} \mathrm{~s}^{-1}$-wide emission 
component on top of one $300-400 \mathrm{~km} \mathrm{~s}^{-1}$-wide. The overall line never appears narrow and symmetric, like in WTTSs: CTTSs-like line emission is present at all phases of both binaries. As is the case with $\mathrm{H} \alpha$ (Basri et al. 1997), the formation of the line survives the lack of the stable long-lived circumstellar disks in the systems.

For a given phase at each orbit there is large stochastic variability in all parts of the line, with the narrow center of the line being more variable than the wings. There is not a definite relationship between phase and line shape.

We decompose the $\mathrm{C}$ IV lines in narrow and broad Gaussians and explore their behavior with phase. For UZ Tau E, the average width of the $\mathrm{NC}\left(\overline{\mathrm{FWHM}}_{\mathrm{NC}}=50 \mathrm{~km} \mathrm{~s}^{-1}\right)$ is significantly smaller than the single CTTS average $\left(\overline{\mathrm{FWHM}}_{\mathrm{NC}}=118 \mathrm{~km} \mathrm{~s}^{-1}\right)$ and it can disappear completely at some phases. The system is observed from a low-inclination angle and so it is unlikely that these disappearances are due to stellar rotation. We find that for both stars, the center of the BC is blueshifted to negative velocities on average, unlike what is generally observed for single CTTSs (Figure 9).

We do not find any correlations between the orbital phase and width or velocity of the lines, for either the broad or narrow components, in any of the systems. Furthermore, we do not find any correlations between any NC or BC Gaussian parameter (flux, width, velocity) and the separation between the stars or the velocity difference between the primary and the secondary.

The data presented here do not allow us to detect line splitting in NCs of DQ Tau, even if both stars were contributing equally to the $\mathrm{C}$ IV profile. However, in the case of UZ Tau E we should be able to detect a splitting of the NC at phase 0.2 . The fact that we do not leads us to conclude that the observed emission should be dominated by one of the stars. Supporting this conclusion we find that the NC velocity is correlated with the velocity of the primary for UZ Tau E (but not for DQ Tau.)

From the results presented here, we believe that, while the $\mathrm{BC}$ in both stars is directly powered by accretion, other processes, such as flares in the stellar magnetospheres and/or enhanced activity in the upper atmosphere, are responsible for the NC. This idea is supported by the large variability of the $\mathrm{NC}$ and the lack of correlation with the NUV continuum (which is a direct accretion indicator). Consistent with this idea, for UZ Tau E the NC luminosities (Figure 8) and widths ( 7 RMSs smaller than in single CTTSs) are comparable to those in WTTSs. DQ Tau has larger NC fluxes and widths, maybe as the result of contributions from both members. We note that the stars do not come as close to each other in UZ Tau E $\left(24 R_{\odot}\right)$ as in DQ Tau $\left(13 R_{\odot}\right)$, likely resulting in a less extreme magnetic environment in the former.

If the observed $\mathrm{C}$ IV NC line is the result of flares produced by interacting magnetospheres, we would expect the NC to be correlated with phase. This is not observed in our data set. The stochastic nature of the flare generation probably means that we need to have a much larger data set before we can observe this correlation, if present. For DQ Tau, millimeter flares at periastron have been detected at a rate of once per orbit (Salter et al. 2008, 2010). These are consistent with synchrotron emission due to magnetic reconnection events in miss-aligned magnetospheres. For UZ Tau E, no submillimeter flares have been detected (Kóspál et al. 2011): very high-cadence monitoring campaigns may be necessary to detect them.
Bary \& Petersen (2014) argue that large increases in the infrared accretion indicators of DQ Tau at phases $0.4-0, .5$ are due to flares. However, the stochastic nature of the accretion process will result in behaviors (explosive increases) that are similar to those of flares, and the flare interpretation by Bary $\&$ Petersen (2014) based on individual events may not be correct.

For the targets considered here, the $\mathrm{C}$ IV BC is directly powered by accretion, as suggested by the correlation with the NUV continuum. If produced in an incoming flow, we would naively expect the line centroid to be redshifted. This is not what is observed (Figure 9). However, as was discussed in A13, the geometry of the accretion region is complex (see for example Donati et al. 2011) and turbulence and projection effects in the shock region may obscure the redshift.

Time-domain studies of young binaries provide us with novel tools to understand star formation. It is clear that many questions remain, which can be addressed with larger or more finely tuned data sets. In particular, we need to obtain additional UV observations close to periastron, and/or at equally spaced points in velocity difference or distance between the stars.

This work is based on observations made with the NASA/ ESA Hubble Space Telescope. Support for this work was provided by NASA through grant number xxx from the Space Telescope Science Institute (STScI), which is operated by the Association of Universities for Research in Astronomy, Inc (AURA) under NASA contract NAS 5-26555. Special thanks go to Steve Penton (STScI) and Kevin France (CASAColorado) for extensive discussions of the errors associated with $\mathrm{COS}$ pointing.

Facility: HST (COS, STIS).

\section{REFERENCES}

Alencar, S. H. P., Melo, C. H. F., Dullemond, C. P., et al. 2003, A\&A, 409, 1037

Ardila, D. R., Basri, G., Walter, F. M., Valenti, J. A., \& Johns-Krull, C. M. 2002, ApJ, 567, 1013

Ardila, D. R., Herczeg, G. J., Gregory, S. G., et al. 2013, ApJS, 207, 1

Artymowicz, P., \& Lubow, S. H. 1996, ApJL, 467, L77

Bary, J. S., \& Petersen, M. S. 2014, ApJ, 792, 64

Basri, G., Johns-Krull, C. M., \& Mathieu, R. D. 1997, AJ, 114, 781

Beck, T. L., Bary, J. S., Dutrey, A., et al. 2012, ApJ, 754, 72

Bertout, C. 2007, in IAU Symp. 243, Star-Disk Interaction in Young Stars, ed. J. Bouvier \& I. Appenzeller (Cambridge: Cambridge Univ. Press),

Boden, A. F., Akeson, R. L., Sargent, A. I., et al. 2009, ApJL, 696, L111

Carr, J. S., Mathieu, R. D., \& Najita, J. R. 2001, ApJ, 551, 454

Cox, A. N. 2000, Allen's Astrophysical Quantities (4th ed.; New York: AIP) Danforth, C. W., Keeney, B. A., Stocke, J. T., Shull, J. M., \& Yao, Y. 2010, ApJ, 720, 976

Dommanget, J., \& Nys, O. 2002, yCat, 1274, 0

Donati, J.-F., Gregory, S. G., Montmerle, T., et al. 2011, MNRAS, 417, 1747 Duchêne, G., \& Kraus, A. 2013, ARA\&A, 51, 269

Dutrey, A., Guilloteau, S., Duvert, G., et al. 1996, A\&A, 309, 493

Furlan, E., Luhman, K. L., Espaillat, C., et al. 2011, ApJS, 195, 3

Ghavamian, P., Froning, C., Osterman, S., Keyes, C. D., \& Sahnow, D. 2010, COS FUV External Spectroscopic Performance, STScI Instrument Science Report COS 2010-09(v1)

Goudfrooij, P., Burgh, E., Aliosi, A., et al. 2010, ISR 2010-10: SMOV: COS NUV Imaging Performance, STScI Instrument Science Report COS 201009(v2)

Green, J. C., Froning, C. S., Osterman, S., et al. 2012, ApJ, 744, 60

Gregory, S. G., Donati, J.-F., Morin, J., et al. 2012, ApJ, 755, 97

Guilloteau, S., Dutrey, A., Piétu, V., \& Boehler, Y. 2011, A\&A, 529, A105

Gullbring, E., Hartmann, L., Briceno, C., \& Calvet, N. 1998, ApJ, 492, 323

Günther, H. M., \& Schmitt, J. H. M. M. 2008, A\&A, 481, 735

Günther, R., \& Kley, W. 2002, A\&A, 387, 550 
Harris, R. J., Andrews, S. M., Wilner, D. J., \& Kraus, A. L. 2012, ApJ, 751,115

Hartigan, P., Edwards, S., \& Pierson, R. 2004, ApJ, 609, 261

Herbst, W., Herbst, D. K., Grossman, E. J., \& Weinstein, D. 1994, AJ, 108, 1906

Herczeg, G. J., \& Hillenbrand, L. A. 2014, ApJ, 786, 97

Holman, M. J., \& Wiegert, P. A. 1999, AJ, 117, 621

Ingleby, L., Calvet, N., Bergin, E., et al. 2009, ApJL, 703, L137

Jensen, E. L. N., Dhital, S., Stassun, K. G., et al. 2007, AJ, 134, 241

Jensen, E. L. N., Koerner, D. W., \& Mathieu, R. D. 1996, AJ, 111, 2431

Kenyon, S. J., \& Hartmann, L. 1995, ApJS, 101, 117

Kóspál, Á, Salter, D. M., Hogerheijde, M. R., Moór, A., \& Blake, G. A. 2011, A\&A, 527, A96

Lamzin, S. A. 1995, A\&A, 295, L20

Loinard, L., Torres, R. M., Mioduszewski, A. J., et al. 2007, ApJ, 671, 546

Martín, E. L., Magazzù, A., Delfosse, X., \& Mathieu, R. D. 2005, A\&A, 429, 939
Massa, D. 2013, Cosmic Origins Spectrograph Instrument Handbook (New York: Springer)

Mathieu, R. D., Martin, E. L., \& Magazzu, A. 1996, in Bulletin of the American Astronomical Society, Vol. 28, American Astronomical Society Meeting Abstract \#188, 920

Mathieu, R. D., Stassun, K., Basri, G., et al. 1997, AJ, 113, 1841

Mayama, S., Tamura, M., Hanawa, T., et al. 2010, Sci, 327, 306

Osterman, S., Green, J., Froning, C., et al. 2011, Ap\&SS, 335, 257

Prato, L., Simon, M., Mazeh, T., Zucker, S., \& McLean, I. S. 2002, ApJL, 579, L99

Salter, D. M., Hogerheijde, M. R., \& Blake, G. A. 2008, A\&A, 492, L21

Salter, D. M., Kóspál, Á, Getman, K. V., et al. 2010, A\&A, 521, A32

Simon, M., Dutrey, A., \& Guilloteau, S. 2001, ApJ, 545, 1034

Stempels, H. C., \& Gahm, G. F. 2004, A\&A, 421, 1159

Torres, R. M., Loinard, L., Mioduszewski, A. J., \& Rodríguez, L. F. 2009, ApJ, 698, 242

Yang, H., Herczeg, G. J., Linsky, J. L., et al. 2012, ApJ, 744, 121 